\title{
An iterative algorithm for system of generalized equilibrium problems and fixed point problem
}

\section{Abdellah Bnouhachem*}

\section{"Correspondence:}

babedallah@yahoo.com

School of Management Science and Engineering, Nanjing University,

Nanjing, 210093, P.R. China

ENSA, Ibn Zohr University, BP 1136,

Agadir, Morocco

\section{Springer}

\begin{abstract}
In this paper, we propose an iterative algorithm for finding a common solution of a system of generalized equilibrium problems and a fixed point problem of strictly pseudo-contractive mapping in the setting of real Hilbert spaces. We prove the strong convergence of the sequence generated by the proposed method to a common solution of a system of generalized equilibrium problems and a hierarchical fixed point problem. Preliminary numerical experiments are included to verify the theoretical assertions of the proposed method. The iterative algorithm and results presented in this paper generalize, unify, and improve the previously known results of this area.
\end{abstract}

MSC: 49J30; 47H09; 47J20

Keywords: system of generalized equilibrium problems; variational inequality; hierarchical fixed point problem; fixed point problem; strictly pseudo-contractive mapping

\section{Introduction}

Let $H$ be a real Hilbert space, whose inner product and norm are denoted by $\langle\cdot, \cdot\rangle$ and $\|\cdot\|$. Let $C$ be a nonempty closed convex subset of $H$. Recently, Ceng and Yao [1] considered the following system of generalized equilibrium problems, which involves finding $\left(x^{*}, y^{*}\right) \in$ $C \times C$ :

$$
\begin{cases}F_{1}\left(x^{*}, x\right)+\left\langle B_{1} y^{*}, x-x^{*}\right\rangle+\frac{1}{\mu_{1}}\left\langle x^{*}-y^{*}, x-x^{*}\right\rangle \geq 0 ; & \forall x \in C \text { and } \mu_{1}>0, \\ F_{2}\left(y^{*}, y\right)+\left\langle B_{2} x^{*}, y-y^{*}\right\rangle+\frac{1}{\mu_{2}}\left\langle y^{*}-x^{*}, y-y^{*}\right\rangle \geq 0 ; & \forall y \in C \text { and } \mu_{2}>0,\end{cases}
$$

where $F_{i}: C \times C \rightarrow H$ is two bifunctions and $B_{i}: C \rightarrow H$ is a nonlinear mapping for each $i=1,2$. The solution set of (1.1) is denoted by $\Omega$.

If $F_{1}=F_{2}=F, B_{1}=B_{2}=B$, and $x^{*}=y^{*}$, then problem (1.1) becomes the following generalized equilibrium problem: Finding $x \in C$ such that

$$
F(x, y)+\langle B x, y-x\rangle \geq 0, \quad \forall y \in C,
$$

which was studied by Takahashi and Takahashi [2]. Inspired by the work of Takahashi and Takahashi [2], and Ceng et al. [3], Ceng et al. [4] introduced and analyzed an iterative scheme for finding the approximate solutions of the generalized equilibrium problem 
(1.2), a system of general generalized equilibrium problems (1.1) and a fixed point problem of a nonexpansive mapping in a Hilbert space. Under appropriate conditions, they proved that the sequence converges strongly to a common solution of these three problems. Recently, Ansari [5] studied the existence of solutions of equilibrium problems in the setting of metric spaces. Inspired by the method in [6], Latif et al. [7] introduced and analyzed an iterative algorithm by the hybrid iterative method for finding a solution of the system of generalized equilibrium problems with constraints of several problems: a generalized mixed equilibrium problem, finitely many variational inclusions, and the common fixed point problem of an asymptotically strict pseudo-contractive mapping in the intermediate sense and infinitely many nonexpansive mappings in a real Hilbert space. Under mild conditions, they proved the weak convergence of this iterative algorithm.

If $F_{1}=F_{2}=0$, then problem (1.1) reduces to the following general system of variational inequalities, which involves finding $\left(x^{*}, y^{*}\right) \in C \times C$ :

$$
\begin{cases}\left\langle\mu_{1} B_{1} y^{*}+x^{*}-y^{*}, x-x^{*}\right\rangle \geq 0 ; & \forall x \in C \text { and } \mu_{1}>0 \\ \left\langle\mu_{2} B_{2} x^{*}+y^{*}-x^{*}, x-y^{*}\right\rangle \geq 0 ; & \forall x \in C \text { and } \mu_{2}>0\end{cases}
$$

this problem was considered and investigated by Ceng et al. [3]. As pointed out in [8] that the system of variational inequalities is used as a tool to study the Nash equilibrium problem; see, for example, [9-11] and the references therein.

If $F_{1}=F_{2}=0$, and $B_{1}=B_{2}=B$, then problem (1.1) reduces to finding $\left(x^{*}, y^{*}\right) \in C \times C$ such that

$$
\begin{cases}\left\langle\mu_{1} B y^{*}+x^{*}-y^{*}, x-x^{*}\right\rangle \geq 0 ; & \forall x \in C \text { and } \mu_{1}>0 \\ \left\langle\mu_{2} B x^{*}+y^{*}-x^{*}, x-y^{*}\right\rangle \geq 0 ; & \forall x \in C \text { and } \mu_{2}>0\end{cases}
$$

which has been introduced and studied by Verma $[12,13]$.

If $x^{*}=y^{*}$ and $\mu_{1}=\mu_{2}$, then problem (1.4) collapses to the classical variational inequality, finding $x^{*} \in C$ such that

$$
\left\langle B x^{*}, x-x^{*}\right\rangle \geq 0, \quad \forall x \in C .
$$

The theory of variational inequalities emerged as a rapidly growing area of research because of its applications in nonlinear analysis, optimization, economics, game theory; see for example [14-17]. For recent applications, numerical techniques, and physical formulation, see [1-50].

The fixed point problem for the mapping $T$ is to find $x \in C$ such that

$$
T x=x .
$$

We denote by $F(T)$ the set of solutions of (1.5). It is well known that $F(T)$ is closed and convex, and $P_{F}(T)$ is well defined (see [19]).

Let $S: C \rightarrow H$ be a nonexpansive mapping, that is, $\|S x-S y\| \leq\|x-y\|$ for all $x, y \in C$. The hierarchical fixed point problem is to find $x \in F(T)$ such that

$$
\langle x-S x, y-x\rangle \geq 0, \quad \forall y \in F(T) .
$$


It is linked with some monotone variational inequalities and convex programming problems; see [20]. Various methods have been proposed to solve (1.6); see, for example, [2135]. By combining Korpelevich's extragradient method and the viscosity approximation method, Ceng et al. [36] introduced and analyzed implicit and explicit iterative schemes for computing a common element of the set of fixed points of a nonexpansive mapping and the set of solutions of the variational inequality for an $\alpha$-inverse strongly monotone mapping in a Hilbert space. Under suitable assumptions, they proved the strong convergence of the sequences generated by the proposed schemes. In 2010, Yao et al. [20] introduced the following strong convergence iterative algorithm to solve problem (1.6):

$$
\begin{aligned}
& y_{n}=\beta_{n} S x_{n}+\left(1-\beta_{n}\right) x_{n}, \\
& x_{n+1}=P_{C}\left[\alpha_{n} f\left(x_{n}\right)+\left(1-\alpha_{n}\right) T y_{n}\right], \quad \forall n \geq 0,
\end{aligned}
$$

where $f: C \rightarrow H$ is a contraction mapping and $\left\{\alpha_{n}\right\}$ and $\left\{\beta_{n}\right\}$ are two sequences in $(0,1)$. Under some certain restrictions on parameters, Yao et al. proved that the sequence $\left\{x_{n}\right\}$ generated by (1.7) converges strongly to $z \in F(T)$, which is the unique solution of the following variational inequality:

$$
\langle(I-f) z, y-z\rangle \geq 0, \quad \forall y \in F(T) .
$$

In 2011, Ceng et al. [37] investigated the following iterative method:

$$
x_{n+1}=P_{C}\left[\alpha_{n} \rho U\left(x_{n}\right)+\left(I-\alpha_{n} \mu F\right)\left(T\left(y_{n}\right)\right)\right], \quad \forall n \geq 0,
$$

where $U$ is a Lipschitzian mapping, and $F$ is a Lipschitzian and strongly monotone mapping. They proved that under some approximate assumptions on the operators and parameters, the sequence $\left\{x_{n}\right\}$ generated by (1.9) converges strongly to the unique solution of the variational inequality

$$
\langle\rho U(z)-\mu F(z), x-z\rangle \geq 0, \quad \forall x \in \operatorname{Fix}(T) .
$$

Very recently, Wang and $\mathrm{Xu}[38]$ investigated an iterative method for a hierarchical fixed point problem by

$$
\begin{aligned}
& y_{n}=\beta_{n} S x_{n}+\left(1-\beta_{n}\right) x_{n}, \\
& x_{n+1}=P_{C}\left[\alpha_{n} \rho U\left(x_{n}\right)+\left(I-\alpha_{n} \mu F\right)\left(T\left(y_{n}\right)\right)\right], \quad \forall n \geq 0,
\end{aligned}
$$

where $S: C \rightarrow C$ is a nonexpansive mapping. They proved that under some approximate assumptions on the operators and parameters, the sequence $\left\{x_{n}\right\}$ generated by (1.11) converges strongly to the unique solution of the variational inequality (1.10). In 2014, Ansari et al. [39] presented a hybrid iterative algorithm for computing a fixed point of a pseudocontractive mapping and for finding a solution of triple hierarchical variational inequality in the setting of real Hilbert space. Under very appropriate conditions, they proved that the sequence generated by the proposed algorithm converges strongly to a fixed point which is also a solution of this triple hierarchical variational inequality. 
In this paper, motivated by the work of Ceng et al. [4], Yao et al. [20], Bnouhachem [33, $34]$ and by the recent work going in this direction, we give an iterative method for finding the approximate element of the common set of solutions of (1.1) and (1.6) in real Hilbert space. We establish a strong convergence theorem based on this method. In order to verify the theoretical assertions and to compare the numerical results between the system of generalized equilibrium problems and the generalized equilibrium problems, an example is given. Our results can be viewed as significant extensions of the previously known results.

\section{Preliminaries}

We present some definitions which will be used in the sequel.

Definition 2.1 A mapping $T: C \rightarrow H$ is said to be $k$-Lipschitz continuous if there exists a constant $k>0$ such that

$$
\|T x-T y\| \leq k\|x-y\|, \quad \forall x, y \in C .
$$

- If $k=1$, then $T$ is called nonexpansive.

- If $k \in(0,1)$, then $T$ is called a contraction.

Definition 2.2 A mapping $T: C \rightarrow H$ is said to be

(a) strongly monotone if there exists an $\alpha>0$ such that

$$
\langle T x-T y, x-y\rangle \geq \alpha\|x-y\|^{2}, \quad \forall x, y \in C ;
$$

(b) $\alpha$-inverse strongly monotone if there exists an $\alpha>0$ such that

$$
\langle T x-T y, x-y\rangle \geq \alpha\|T x-T y\|^{2}, \quad \forall x, y \in C ;
$$

(c) a $k$-strict pseudo-contraction, if there exists a constant $0 \leq k<1$ such that

$$
\|T x-T y\|^{2} \leq\|x-y\|^{2}+k\|(I-T) x-(I-T) y\|^{2}, \quad \forall x, y \in C .
$$

Assumption 2.1 [42] Let $F: C \times C \rightarrow \mathbb{R}$ be a bifunction satisfying the following assumptions:

$\left(\mathrm{A}_{1}\right) \quad F(x, x)=0, \forall x \in C$;

$\left(\mathrm{A}_{2}\right) F$ is monotone, i.e., $F(x, y)+F(y, x) \leq 0, \forall x, y \in C$;

$\left(\mathrm{A}_{3}\right)$ for each $x, y, z \in C, \lim _{t \rightarrow 0} F(t z+(1-t) x, y) \leq F(x, y)$;

$\left(\mathrm{A}_{4}\right)$ for each $x \in C, y \rightarrow F(x, y)$ is convex and lower semicontinuous.

We list some fundamental lemmas that are useful in the consequent analysis.

Lemma 2.1 [43] Let $C$ be a nonempty closed convex subset of $H$. Let $F: C \times C \rightarrow \mathbb{R}$ satisfies $\left(\mathrm{A}_{1}\right)-\left(\mathrm{A}_{4}\right)$. Assume that for $r>0$ and $\forall x \in H$, define a mapping $T_{r}: H \rightarrow C$ as follows:

$$
T_{r}(x)=\left\{z \in C: F(z, y)+\frac{1}{r}\langle y-z, z-x\rangle \geq 0, \forall y \in C\right\} .
$$


Then the following hold:

(i) $T_{r}$ is nonempty and single-valued;

(ii) $T_{r}$ is firmly nonexpansive, i.e.,

$$
\left\|T_{r}(x)-T_{r}(y)\right\|^{2} \leq\left\langle T_{r}(x)-T_{r}(y), x-y\right\rangle, \quad \forall x, y \in H ;
$$

(iii) $F\left(T_{r}\right)=\mathrm{EP}(F)$;

(iv) $\mathrm{EP}(F)$ is closed and convex.

Lemma 2.2 [4] Let $F_{1}, F_{2}: C \times C \rightarrow \mathbb{R}$ be two bifunctions satisfying $\left(\mathrm{A}_{1}\right)-\left(\mathrm{A}_{4}\right)$. For any $\left(x^{*}, y^{*}\right) \in C \times C,\left(x^{*}, y^{*}\right)$ is a solution of (1.1) if and only if $x^{*}$ is a fixed point of the mapping $Q: C \rightarrow C$ defined by

$$
Q(x)=T_{\mu_{1}}^{F_{1}}\left[T_{\mu_{2}}^{F_{2}}\left[x-\mu_{2} B_{2} x\right]-\mu_{1} B_{1} T_{\mu_{2}}^{F_{2}}\left[x-\mu_{2} B_{2} x\right]\right], \quad \forall x \in C,
$$

where $y^{*}=T_{\mu_{2}}^{F_{2}}\left[x^{*}-\mu_{2} B_{2} x^{*}\right], \mu_{i} \in\left(0,2 \theta_{i}\right)$, and $B_{i}: C \rightarrow C$ is a $\theta_{i}$-inverse strongly monotone mapping for each $i=1,2$.

Lemma 2.3 [44] Let $C$ be a nonempty closed convex subset of a real Hilbert space $H$.

If $T: C \rightarrow C$ is a nonexpansive mapping with $\operatorname{Fix}(T) \neq \emptyset$, then the mapping $I-T$ is demiclosed at 0 , i.e., if $\left\{x_{n}\right\}$ is a sequence in $C$ that weakly converges to $x$, and if $\left\{(I-T) x_{n}\right\}$ converges strongly to 0 , then $(I-T) x=0$.

Lemma 2.4 [37] Let $U: C \rightarrow H$ be a $\tau$-Lipschitzian mapping, and let $F: C \rightarrow H$ be a $k$-Lipschitzian and $\eta$-strongly monotone mapping, then for $0 \leq \rho \tau<\mu \eta, \mu F-\rho U$ is $\mu \eta$ $\rho \tau$-strongly monotone, i.e.,

$$
\langle(\mu F-\rho U) x-(\mu F-\rho U) y, x-y\rangle \geq(\mu \eta-\rho \tau)\|x-y\|^{2}, \quad \forall x, y \in C .
$$

Lemma 2.5 [45] Let $C$ be a nonempty closed convex subset of a real Hilbert space $H$, and $S$ : $C \rightarrow H$ be a k-strict pseudo-contraction mapping. Define $B: C \rightarrow H$ by $B x=\lambda S x+(1-\lambda) x$ for all $x \in C$. Then as $\lambda \in[k, 1), B$ is a nonexpansive mapping such that $F(B)=F(S)$.

Lemma 2.6 [46] Let $H$ be a real Hilbert space, $T: C \rightarrow H$ be a k-Lipschitzian and $\eta$-strongly monotone operator. Let $0<\mu<\frac{2 \eta}{k^{2}}$, let $W=I-\lambda \mu T$ and $\mu\left(\eta-\frac{\mu k^{2}}{2}\right)=\tau$, then for $0<\lambda<\min \left\{1, \frac{1}{\tau}\right\}, W$ is a contraction with a constant $1-\lambda \tau$, that is,

$$
\|W x-W y\| \leq(1-\lambda \tau)\|x-y\|, \quad \forall x, y \in C .
$$

Lemma 2.7 [47] Let $\left\{x_{n}\right\},\left\{y_{n}\right\}$ be bounded sequences in a Banach space $E$ and $\left\{\beta_{n}\right\}$ be a sequence in $[0,1]$ with $0<\liminf _{n \rightarrow \infty} \beta_{n}<\limsup _{n \rightarrow \infty} \beta_{n}<1$.

Suppose $x_{n+1}=\beta_{n} x_{n}+\left(1-\beta_{n}\right) y_{n}, \forall n \geq 0$ and $\lim \sup _{n \rightarrow \infty}\left(\left\|y_{n+1}-y_{n}\right\|-\left\|x_{n+1}-x_{n}\right\|\right) \leq 0$. Then $\lim _{n \rightarrow \infty}\left\|y_{n}-x_{n}\right\|=0$.

Lemma 2.8 [48] Assume $\left\{a_{n}\right\}$ is a sequence of nonnegative real numbers such that

$$
a_{n+1} \leq\left(1-v_{n}\right) a_{n}+\delta_{n}
$$


where $\left\{v_{n}\right\}$ is a sequence in $(0,1)$ and $\delta_{n}$ is a sequence such that

(1) $\sum_{n=1}^{\infty} v_{n}=\infty$;

(2) $\lim \sup _{n \rightarrow \infty} \delta_{n} / v_{n} \leq 0$ or $\sum_{n=1}^{\infty}\left|\delta_{n}\right|<\infty$.

Then $\lim _{n \rightarrow \infty} a_{n}=0$.

Lemma 2.9 [49] Let C be a closed convex subset of H. Let $\left\{x_{n}\right\}$ be a bounded sequence in $H$. Assume that

(i) the weak $w$-limit set $w_{w}\left(x_{n}\right) \subset C$ where $w_{w}\left(x_{n}\right)=\left\{x: x_{n_{i}} \rightarrow x\right\}$;

(ii) for each $z \in C, \lim _{n \rightarrow \infty}\left\|x_{n}-z\right\|$ exists.

Then $\left\{x_{n}\right\}$ is weakly convergent to a point in $C$.

Lemma 2.10 [50] Let $H$ be a real Hilbert space. Then the following inequality holds:

$$
\|x+y\|^{2} \leq\|x\|^{2}+2\langle y, x+y\rangle, \quad \forall x, y \in H
$$

\section{The proposed method and some properties}

In this section, we suggest and analyze our method for finding the common solutions of the system of the generalized equilibrium problem (1.1) and the hierarchical fixed point problem (1.6). Let $C$ be a nonempty closed convex subset of a real Hilbert space $H$. Let $F_{1}, F_{2}: C \times C \rightarrow \mathbb{R}$ be two bifunctions satisfying $\left(\mathrm{A}_{1}\right)-\left(\mathrm{A}_{4}\right)$. Let $B_{i}: C \rightarrow H$ be a $\theta_{i}$-inverse strongly monotone mapping for each $i=1,2$, and let $S: C \rightarrow H$ be a $\sigma$-strict pseudocontraction mapping such that $\Omega \cap F(S) \neq \emptyset$. Let $T: C \rightarrow C$ be a $k$-Lipschitzian mapping and be $\eta$-strongly monotone, and let $f: C \rightarrow C$ be a $\tau$-Lipschitzian mapping.

Algorithm 3.1 For an arbitrarily given $x_{0} \in C$, let the iterative sequences $\left\{x_{n}\right\},\left\{y_{n}\right\}$, and $\left\{z_{n}\right\}$ be generated by

$$
\left\{\begin{array}{l}
z_{n}=T_{\mu_{1}}^{F_{1}}\left[T_{\mu_{2}}^{F_{2}}\left[x_{n}-\mu_{2} B_{2} x_{n}\right]-\mu_{1} B_{1} T_{\mu_{2}}^{F_{2}}\left[x_{n}-\mu_{2} B_{2} x_{n}\right]\right] ; \\
y_{n}=\beta_{n} S z_{n}+\left(1-\beta_{n}\right) z_{n} ; \\
x_{n+1}=\alpha_{n} \rho f\left(x_{n}\right)+\gamma_{n} x_{n}+\left(\left(1-\gamma_{n}\right) I-\alpha_{n} \mu T\right)\left(y_{n}\right), \quad \forall n \geq 0,
\end{array}\right.
$$

where $\mu_{i} \in\left(0,2 \theta_{i}\right)$ for each $i=1,2$. Suppose the parameters satisfy $0<\mu<\frac{2 \eta}{k^{2}}, 0 \leq \rho<$ $v / \tau$, where $v=\mu\left(\eta-\frac{\mu k^{2}}{2}\right)$. Also $\left\{\gamma_{n}\right\},\left\{\alpha_{n}\right\}$, and $\left\{\beta_{n}\right\}$ are sequences in $(0,1)$ satisfying the following conditions:

(a) $0<\liminf _{n \rightarrow \infty} \gamma_{n}<\limsup _{n \rightarrow \infty} \gamma_{n}<1$

(b) $\lim _{n \rightarrow \infty} \alpha_{n}=0$ and $\sum_{n=1}^{\infty} \alpha_{n}=\infty$;

(c) $\left\{\beta_{n}\right\} \subset[\sigma, 1)$ and $\lim _{n \rightarrow \infty} \beta_{n}=\beta<1$.

If $F_{1}=F_{2}=F, B_{1}=B_{2}=B$, and $\mu_{1}=\mu_{2}=r$, then Algorithm 3.1 reduces to Algorithm 3.2 for finding the common solutions of the generalized equilibrium problem (1.2) and the hierarchical fixed point problem (1.6).

Algorithm 3.2 For an arbitrarily given $x_{0} \in C$ arbitrarily, let the iterative sequences $\left\{u_{n}\right\}$, $\left\{x_{n}\right\},\left\{y_{n}\right\}$, and $\left\{z_{n}\right\}$ be generated by

$$
\left\{\begin{array}{l}
F\left(z_{n}, y\right)+\left\langle B x_{n}, y-z_{n}\right\rangle+\frac{1}{r}\left\langle y-z_{n}, z_{n}-x_{n}\right\rangle \geq 0, \quad \forall y \in C ; \\
y_{n}=\beta_{n} S z_{n}+\left(1-\beta_{n}\right) z_{n} \\
x_{n+1}=\alpha_{n} \rho f\left(x_{n}\right)+\gamma_{n} x_{n}+\left(\left(1-\gamma_{n}\right) I-\alpha_{n} \mu T\right)\left(y_{n}\right), \quad \forall n \geq 0 .
\end{array}\right.
$$


Suppose that the parameters satisfy $0<\mu<\frac{2 \eta}{k^{2}}, 0 \leq \rho \tau<\nu$, where $v=1-\sqrt{1-\mu\left(2 \eta-\mu k^{2}\right)}$. Also $\left\{\gamma_{n}\right\},\left\{\alpha_{n}\right\}$, and $\left\{\beta_{n}\right\}$ are sequences in $(0,1)$ satisfying the following conditions:

(a) $0<\liminf _{n \rightarrow \infty} \gamma_{n}<\limsup _{n \rightarrow \infty} \gamma_{n}<1$;

(b) $\lim _{n \rightarrow \infty} \alpha_{n}=0$ and $\sum_{n=1}^{\infty} \alpha_{n}=\infty$;

(c) $\left\{\beta_{n}\right\} \subset[\sigma, 1)$ and $\lim _{n \rightarrow \infty} \beta_{n}=\beta<1$.

Remark 3.1 If $\rho=\mu=1, \gamma_{n}=0$, and $S z_{n}=S x_{n}$, we obtain an extension and improvement of the method of Yao et al. [20] and Wang and Xu [38] for finding the approximate element of the common set of solutions of a system of generalized equilibrium problem and a hierarchical fixed point problem in a real Hilbert space.

Lemma 3.1 Let $x^{*} \in \Omega \cap F(S)$. Then $\left\{x_{n}\right\},\left\{z_{n}\right\}$, and $\left\{y_{n}\right\}$ are bounded.

Proof Let $x^{*} \in \Omega \cap F(S)$, we have

$$
x^{*}=T_{\mu_{1}}^{F_{1}}\left[y^{*}-\mu_{1} B_{1} y^{*}\right]
$$

where

$$
y^{*}=T_{\mu_{2}}^{F_{2}}\left[x^{*}-\mu_{2} B_{2} x^{*}\right]
$$

We set $v_{n}=T_{\mu_{2}}^{F_{2}}\left[x_{n}-\mu_{2} B_{2} x_{n}\right]$. Since $B_{2}$ is a $\theta_{2}$-inverse strongly monotone mapping, it follows that

$$
\begin{aligned}
\left\|v_{n}-y^{*}\right\|^{2} & =\left\|T_{\mu_{2}}^{F_{2}}\left[x_{n}-\mu_{2} B_{2} x_{n}\right]-T_{\mu_{2}}^{F_{2}}\left[x^{*}-\mu_{2} B_{2} x^{*}\right]\right\|^{2} \\
& \leq\left\|x_{n}-x^{*}-\mu_{2}\left(B_{2} x_{n}-B_{2} x^{*}\right)\right\|^{2} \\
& \leq\left\|x_{n}-x^{*}\right\|^{2}-\mu_{2}\left(2 \theta_{2}-\mu_{2}\right)\left\|B_{2} x_{n}-B_{2} x^{*}\right\|^{2} \\
& \leq\left\|x_{n}-x^{*}\right\|^{2} .
\end{aligned}
$$

Since $B_{i}$ is a $\theta_{i}$-inverse strongly monotone mapping for each $i=1$, 2 , we get

$$
\begin{aligned}
\left\|z_{n}-x^{*}\right\|^{2}= & \| T_{\mu_{1}}^{F_{1}}\left[T_{\mu_{2}}^{F_{2}}\left[x_{n}-\mu_{2} B_{2} x_{n}\right]-\mu_{1} B_{1} T_{\mu_{2}}^{F_{2}}\left[x_{n}-\mu_{2} B_{2} x_{n}\right]\right] \\
& -T_{\mu_{1}}^{F_{1}}\left[T_{\mu_{2}}^{F_{2}}\left[x^{*}-\mu_{2} B_{2} x^{*}\right]-\mu_{1} B_{1} T_{\mu_{2}}^{F_{2}}\left[x^{*}-\mu_{2} B_{2} x^{*}\right]\right] \|^{2} \\
\leq & \| T_{\mu_{2}}^{F_{2}}\left[x_{n}-\mu_{2} B_{2} x_{n}\right]-\mu_{1} B_{1} T_{\mu_{2}}^{F_{2}}\left[x_{n}-\mu_{2} B_{2} x_{n}\right] \\
& -\left(T_{\mu_{2}}^{F_{2}}\left[x^{*}-\mu_{2} B_{2} x^{*}\right]-\mu_{1} B_{1} T_{\mu_{2}}^{F_{2}}\left[x^{*}-\mu_{2} B_{2} x^{*}\right]\right) \|^{2} \\
= & \| T_{\mu_{2}}^{F_{2}}\left[x_{n}-\mu_{2} B_{2} x_{n}\right]-T_{\mu_{2}}^{F_{2}}\left[x^{*}-\mu_{2} B_{2} x^{*}\right] \\
& -\mu_{1}\left(B_{1} T_{\mu_{2}}^{F_{2}}\left[x_{n}-\mu_{2} B_{2} x_{n}\right]-B_{1} T_{\mu_{2}}^{F_{2}}\left[x^{*}-\mu_{2} B_{2} x^{*}\right]\right) \|^{2} \\
\leq & \left\|T_{\mu_{2}}^{F_{2}}\left[x_{n}-\mu_{2} B_{2} x_{n}\right]-T_{\mu_{2}}^{F_{2}}\left[x^{*}-\mu_{2} B_{2} x^{*}\right]\right\|^{2} \\
& -\mu_{1}\left(2 \theta_{1}-\mu_{1}\right)\left\|B_{1} T_{\mu_{2}}^{F_{2}}\left[x_{n}-\mu_{2} B_{2} x_{n}\right]-B_{1} T_{\mu_{2}}^{F_{2}}\left[x^{*}-\mu_{2} B_{2} x^{*}\right]\right\|^{2} \\
\leq & \left\|\left(x_{n}-\mu_{2} B_{2} x_{n}\right)-\left(x^{*}-\mu_{2} B_{2} x^{*}\right)\right\|^{2} \\
& -\mu_{1}\left(2 \theta_{1}-\mu_{1}\right)\left\|B_{1} T_{\mu_{2}}^{F_{2}}\left[x_{n}-\mu_{2} B_{2} x_{n}\right]-B_{1} T_{\mu_{2}}^{F_{2}}\left[x^{*}-\mu_{2} B_{2} x^{*}\right]\right\|^{2} \\
\leq & \left\|x_{n}-x^{*}\right\|^{2}-\mu_{2}\left(2 \theta_{2}-\mu_{2}\right)\left\|B_{2} x_{n}-B_{2} x^{*}\right\|^{2}
\end{aligned}
$$




$$
\begin{aligned}
& -\mu_{1}\left(2 \theta_{1}-\mu_{1}\right)\left\|B_{1} v_{n}-B_{1} y^{*}\right\|^{2} \\
\leq & \left\|x_{n}-x^{*}\right\|^{2} .
\end{aligned}
$$

By Lemma 2.5 and the inequality above, it is easy to show that

$$
\left\|y_{n}-x^{*}\right\| \leq\left\|z_{n}-x^{*}\right\| \leq\left\|x_{n}-x^{*}\right\| .
$$

Next, we prove that the sequence $\left\{x_{n}\right\}$ is bounded. Since $\lim _{n \rightarrow \infty} \alpha_{n}=0$, without loss of generality we can assume that $\alpha_{n} \leq \min \left\{\epsilon, \frac{\epsilon}{\tau}\right\}$ for all $n \geq 1$, where $0<\epsilon<1-\lim \sup _{n \rightarrow \infty} \gamma_{n}$. From (3.1) and (3.4), we have

$$
\begin{aligned}
\left\|x_{n+1}-x^{*}\right\| \leq & \left\|\alpha_{n} \rho f\left(x_{n}\right)+\gamma_{n} x_{n}+\left(\left(1-\gamma_{n}\right) I-\alpha_{n} \mu T\right)\left(y_{n}\right)-x^{*}\right\| \\
= & \| \alpha_{n}\left(\rho f\left(x_{n}\right)-\mu T\left(x^{*}\right)\right)+\gamma_{n}\left(x_{n}-x^{*}\right)+\left(\left(1-\gamma_{n}\right) I-\alpha_{n} \mu T\right)\left(y_{n}\right) \\
& -\left(\left(1-\gamma_{n}\right) I-\alpha_{n} \mu T\right)\left(x^{*}\right) \| \\
\leq & \alpha_{n} \rho \tau\left\|x_{n}-x^{*}\right\|+\alpha_{n}\left\|(\rho f-\mu T) x^{*}\right\|+\gamma_{n}\left\|x_{n}-x^{*}\right\| \\
& +\left\|\left(\left(1-\gamma_{n}\right) I-\alpha_{n} \mu T\right)\left(y_{n}\right)-\left(\left(1-\gamma_{n}\right) I-\alpha_{n} \mu T\right)\left(x^{*}\right)\right\| \\
= & \alpha_{n} \rho \tau\left\|x_{n}-x^{*}\right\|+\alpha_{n}\left\|(\rho f-\mu T) x^{*}\right\|+\gamma_{n}\left\|x_{n}-x^{*}\right\| \\
& +\left(1-\gamma_{n}\right)\left\|\left(I-\frac{\alpha_{n} \mu}{\left(1-\gamma_{n}\right)} T\right)\left(y_{n}\right)-\left(I-\frac{\alpha_{n} \mu}{\left(1-\gamma_{n}\right)} T\right)\left(x^{*}\right)\right\| \\
\leq & \alpha_{n} \rho \tau\left\|x_{n}-x^{*}\right\|+\alpha_{n}\left\|(\rho f-\mu T) x^{*}\right\|+\gamma_{n}\left\|x_{n}-x^{*}\right\| \\
& +\left(1-\gamma_{n}-\alpha_{n} v\right)\left\|y_{n}-x^{*}\right\| \\
\leq & \alpha_{n} \rho \tau\left\|x_{n}-x^{*}\right\|+\alpha_{n}\left\|(\rho f-\mu T) x^{*}\right\|+\gamma_{n}\left\|x_{n}-x^{*}\right\| \\
& +\left(1-\gamma_{n}-\alpha_{n} \nu\right)\left\|x_{n}-x^{*}\right\| \\
= & \alpha_{n} \rho \tau\left\|x_{n}-x^{*}\right\|+\alpha_{n}\left\|(\rho f-\mu T) x^{*}\right\|+\left(1-\alpha_{n} v\right)\left\|x_{n}-x^{*}\right\| \\
= & \left(1-\alpha_{n}(v-\rho \tau)\right)\left\|x_{n}-x^{*}\right\|+\alpha_{n}\left\|(\rho f-\mu T) x^{*}\right\| \\
\leq & \max \left\{\left\|x_{n}-x^{*}\right\|, \frac{1}{v-\rho \tau}\left(\left\|(\rho f-\mu T) x^{*}\right\|\right)\right\},
\end{aligned}
$$

where the third inequality follows from Lemma 2.6 and the fourth inequality follows from (3.4). By induction on $n$, we obtain $\left\|x_{n}-x^{*}\right\| \leq \max \left\{\left\|x_{n}-x^{*}\right\|, \frac{1}{v-\rho \tau}\left(\left\|(\rho f-\mu T) x^{*}\right\|\right)\right\}$, for $n \geq 0$ and $x_{0} \in C$. Hence $\left\{x_{n}\right\}$ is bounded, and consequently we deduce that $\left\{z_{n}\right\},\left\{v_{n}\right\},\left\{y_{n}\right\}$, $\left\{S\left(z_{n}\right)\right\},\left\{T\left(y_{n}\right)\right\}$, and $\left\{f\left(x_{n}\right)\right\}$ are bounded.

Lemma 3.2 Let $x^{*} \in \Omega \cap F(S)$ and $\left\{x_{n}\right\}$ be the sequence generated by Algorithm 3.1. Then we have:

(a) $\lim _{n \rightarrow \infty}\left\|x_{n+1}-x_{n}\right\|=0$.

(b) The weak $w$-limit set $w_{w}\left(x_{n}\right) \subset F(S)\left(w_{w}\left(x_{n}\right)=\left\{x: x_{n_{i}} \rightarrow x\right\}\right)$.

Proof Next, we estimate

$$
\begin{aligned}
\left\|z_{n}-z_{n-1}\right\|^{2}= & \| T_{\mu_{1}}^{F_{1}}\left[T_{\mu_{2}}^{F_{2}}\left[x_{n}-\mu_{2} B_{2} x_{n}\right]-\mu_{1} B_{1} T_{\mu_{2}}^{F_{2}}\left[x_{n}-\mu_{2} B_{2} x_{n}\right]\right] \\
& -T_{\mu_{1}}^{F_{1}}\left[T_{\mu_{2}}^{F_{2}}\left[x_{n-1}-\mu_{2} B_{2} x_{n-1}\right]-\mu_{1} B_{1} T_{\mu_{2}}^{F_{2}}\left[x_{n-1}-\mu_{2} B_{2} x_{n-1}\right]\right] \|^{2}
\end{aligned}
$$




$$
\begin{aligned}
\leq & \| T_{\mu_{2}}^{F_{2}}\left[x_{n}-\mu_{2} B_{2} x_{n}\right]-\mu_{1} B_{1} T_{\mu_{2}}^{F_{2}}\left[x_{n}-\mu_{2} B_{2} x_{n}\right] \\
& -\left(T_{\mu_{2}}^{F_{2}}\left[x_{n-1}-\mu_{2} B_{2} x_{n-1}\right]-\mu_{1} B_{1} T_{\mu_{2}}^{F_{2}}\left[x_{n-1}-\mu_{2} B_{2} x_{n-1}\right]\right) \|^{2} \\
= & \| T_{\mu_{2}}^{F_{2}}\left[x_{n}-\mu_{2} B_{2} x_{n}\right]-T_{\mu_{2}}^{F_{2}}\left[x_{n-1}-\mu_{2} B_{2} x_{n-1}\right] \\
& -\mu_{1}\left(B_{1} T_{\mu_{2}}^{F_{2}}\left[x_{n}-\mu_{2} B_{2} x_{n}\right]-B_{1} T_{\mu_{2}}^{F_{2}}\left[x_{n-1}-\mu_{2} B_{2} x_{n-1}\right]\right) \|^{2} \\
\leq & \left\|T_{\mu_{2}}^{F_{2}}\left[x_{n}-\mu_{2} B_{2} x_{n}\right]-T_{\mu_{2}}^{F_{2}}\left[x_{n-1}-\mu_{2} B_{2} x_{n-1}\right]\right\|^{2} \\
& -\mu_{1}\left(2 \theta_{1}-\mu_{1}\right)\left\|B_{1} T_{\mu_{2}}^{F_{2}}\left[x_{n}-\mu_{2} B_{2} x_{n}\right]-B_{1} T_{\mu_{2}}^{F_{2}}\left[x_{n-1}-\mu_{2} B_{2} x_{n-1}\right]\right\|^{2} \\
\leq & \left\|T_{\mu_{2}}^{F_{2}}\left[x_{n}-\mu_{2} B_{2} x_{n}\right]-T_{\mu_{2}}^{F_{2}}\left[x_{n-1}-\mu_{2} B_{2} x_{n-1}\right]\right\|^{2} \\
\leq & \left\|\left(x_{n}-x_{n-1}\right)-\mu_{2}\left(B_{2} x_{n}-B_{2} x_{n-1}\right)\right\|^{2} \\
\leq & \left\|x_{n}-x_{n-1}\right\|^{2}-\mu_{2}\left(2 \theta_{2}-\mu_{2}\right)\left\|B_{2} x_{n}-B_{2} x_{n-1}\right\|^{2} \\
\leq & \left\|x_{n}-x_{n-1}\right\|^{2} .
\end{aligned}
$$

From (3.1) and (3.5), we have

$$
\begin{aligned}
\left\|y_{n}-y_{n-1}\right\| \leq & \left\|\beta_{n} S z_{n}+\left(1-\beta_{n}\right) z_{n}-\left(\beta_{n-1} S z_{n-1}+\left(1-\beta_{n-1}\right) z_{n-1}\right)\right\| \\
= & \| \beta_{n}\left(S z_{n}-S z_{n-1}\right)+\left(\beta_{n}-\beta_{n-1}\right) S z_{n-1} \\
& \quad+\left(1-\beta_{n}\right)\left(z_{n}-z_{n-1}\right)+\left(\beta_{n-1}-\beta_{n}\right) z_{n-1} \| \\
\leq & \left\|z_{n}-z_{n-1}\right\|+\left|\beta_{n}-\beta_{n-1}\right|\left\|S z_{n-1}-z_{n-1}\right\| \\
\leq & \left\|x_{n-1}-x_{n}\right\|+\left|\beta_{n}-\beta_{n-1}\right|\left\|S z_{n-1}-z_{n-1}\right\| .
\end{aligned}
$$

We define $w_{n}=\frac{x_{n+1}-\gamma_{n} x_{n}}{1-\gamma_{n}}$, which implies that $x_{n+1}=\left(1-\gamma_{n}\right) w_{n}+\gamma_{n} x_{n}$. It follows from (3.6) that

$$
\begin{aligned}
\left\|w_{n+1}-w_{n}\right\| \leq & \frac{\alpha_{n+1}}{1-\gamma_{n+1}}\left\|\rho f\left(x_{n+1}\right)-\mu T\left(y_{n+1}\right)\right\| \\
& +\frac{\alpha_{n}}{1-\gamma_{n}}\left\|\rho f\left(x_{n}\right)-\mu T\left(y_{n}\right)\right\|+\left\|y_{n+1}-y_{n}\right\| \\
\leq & \frac{\alpha_{n+1}}{1-\gamma_{n+1}}\left\|\rho f\left(x_{n+1}\right)-\mu T\left(y_{n+1}\right)\right\| \\
& +\frac{\alpha_{n}}{1-\gamma_{n}}\left\|\rho f\left(x_{n}\right)-\mu T\left(y_{n}\right)\right\|+\left\|x_{n+1}-x_{n}\right\| \\
& +\left|\beta_{n+1}-\beta_{n}\right|\left\|S z_{n}-z_{n}\right\| .
\end{aligned}
$$

Since $\lim _{n \rightarrow \infty} \alpha_{n}=0, \lim _{n \rightarrow \infty} \beta_{n}=\beta$, and $\liminf _{n \rightarrow \infty} \gamma_{n}<\lim \sup _{n \rightarrow \infty} \gamma_{n}<1$, we get

$$
\limsup _{n \rightarrow \infty}\left(\left\|w_{n+1}-w_{n}\right\|-\left\|x_{n+1}-x_{n}\right\|\right) \leq 0
$$

By Lemma 2.7, we have $\lim _{n \rightarrow \infty}\left\|w_{n}-x_{n}\right\|=0$. Since $\left\|x_{n+1}-x_{n}\right\|=\left(1-\gamma_{n}\right)\left\|w_{n}-x_{n}\right\|$, we obtain

$$
\lim _{n \rightarrow \infty}\left\|x_{n+1}-x_{n}\right\|=0
$$


Next, we estimate

$$
\begin{aligned}
\left\|x_{n}-y_{n}\right\| & \leq\left\|x_{n+1}-x_{n}\right\|+\left\|x_{n+1}-y_{n}\right\| \\
& \leq\left\|x_{n+1}-x_{n}\right\|+\left\|x_{n+1}-y_{n}\right\| \\
& \leq\left\|x_{n+1}-x_{n}\right\|+\alpha_{n}\left\|\rho f\left(x_{n}\right)-\mu T\left(y_{n}\right)\right\|+\gamma_{n}\left\|x_{n}-y_{n}\right\|,
\end{aligned}
$$

which implies

$$
\left(1-\gamma_{n}\right)\left\|x_{n}-y_{n}\right\| \leq\left\|x_{n+1}-x_{n}\right\|+\alpha_{n}\left\|\rho f\left(x_{n}\right)-\mu T\left(y_{n}\right)\right\|
$$

Since $\lim _{n \rightarrow \infty} \alpha_{n}=0$ and $\liminf _{n \rightarrow \infty} \gamma_{n}<\limsup \sup _{n \rightarrow \infty} \gamma_{n}<1$, we have

$$
\lim _{n \rightarrow \infty}\left\|x_{n}-y_{n}\right\|=0
$$

Next, we show that $\lim _{n \rightarrow \infty}\left\|z_{n}-x_{n}\right\|=0$. Since $x^{*} \in \Omega \cap F(S)$ by using Lemma 2.10, (3.4), and (3.3), we obtain

$$
\begin{aligned}
\left\|x_{n+1}-x^{*}\right\|^{2}= & \left\|\gamma_{n}\left(x_{n}-x^{*}\right)+\left(1-\gamma_{n}\right)\left(y_{n}-x^{*}\right)+\alpha_{n}\left(\rho f\left(x_{n}\right)-\mu T\left(y_{n}\right)\right)\right\|^{2} \\
\leq & \left\|\gamma_{n}\left(x_{n}-x^{*}\right)+\left(1-\gamma_{n}\right)\left(y_{n}-x^{*}\right)\right\|^{2}+2 \alpha_{n}\left\langle\rho f\left(x_{n}\right)-\mu T\left(y_{n}\right), x_{n+1}-x^{*}\right\rangle \\
\leq & \gamma_{n}\left\|x_{n}-x^{*}\right\|^{2}+\left(1-\gamma_{n}\right)\left\|y_{n}-x^{*}\right\|^{2}+2 \alpha_{n}\left\langle\rho f\left(x_{n}\right)-\mu T\left(y_{n}\right), x_{n+1}-x^{*}\right\rangle \\
\leq & \gamma_{n}\left\|x_{n}-x^{*}\right\|^{2}+\left(1-\gamma_{n}\right)\left\|z_{n}-x^{*}\right\|^{2}+2 \alpha_{n}\left\langle\rho f\left(x_{n}\right)-\mu T\left(y_{n}\right), x_{n+1}-x^{*}\right\rangle \\
\leq & \gamma_{n}\left\|x_{n}-x^{*}\right\|^{2}+\left(1-\gamma_{n}\right)\left\{\left\|x_{n}-x^{*}\right\|^{2}-\mu_{2}\left(2 \theta_{2}-\mu_{2}\right)\left\|B_{2} x_{n}-B_{2} x^{*}\right\|^{2}\right. \\
& \left.-\mu_{1}\left(2 \theta_{1}-\mu_{1}\right)\left\|B_{1} v_{n}-B_{1} y^{*}\right\|^{2}\right\}+2 \alpha_{n}\left\langle\rho f\left(x_{n}\right)-\mu T\left(y_{n}\right), x_{n+1}-x^{*}\right\rangle \\
= & \left\|x_{n}-x^{*}\right\|^{2}-\left(1-\gamma_{n}\right)\left\{\mu_{2}\left(2 \theta_{2}-\mu_{2}\right)\left\|B_{2} x_{n}-B_{2} x^{*}\right\|^{2}\right. \\
& \left.+\mu_{1}\left(2 \theta_{1}-\mu_{1}\right)\left\|B_{1} v_{n}-B_{1} y^{*}\right\|^{2}\right\} \\
& +2 \alpha_{n}\left\langle\rho f\left(x_{n}\right)-\mu T\left(y_{n}\right), x_{n+1}-x^{*}\right\rangle,
\end{aligned}
$$

which implies that

$$
\begin{aligned}
& \left(1-\gamma_{n}\right)\left\{\mu_{2}\left(2 \theta_{2}-\mu_{2}\right)\left\|B_{2} x_{n}-B_{2} x^{*}\right\|^{2}+\mu_{1}\left(2 \theta_{1}-\mu_{1}\right)\left\|B_{1} v_{n}-B_{1} y^{*}\right\|^{2}\right\} \\
& \quad \leq\left\|x_{n}-x^{*}\right\|^{2}-\left\|x_{n+1}-x^{*}\right\|^{2}+2 \alpha_{n}\left\langle\rho f\left(x_{n}\right)-\mu T\left(y_{n}\right), x_{n+1}-x^{*}\right\rangle \\
& \quad \leq\left(\left\|x_{n}-x^{*}\right\|+\left\|x_{n+1}-x^{*}\right\|\right)\left\|x_{n+1}-x_{n}\right\|+2 \alpha_{n}\left\langle\rho f\left(x_{n}\right)-\mu T\left(y_{n}\right), x_{n+1}-x^{*}\right\rangle .
\end{aligned}
$$

Since $\lim \sup _{n \rightarrow \infty} \gamma_{n}<1,2 \theta_{1}-\mu_{1}>0,2 \theta_{2}-\mu_{2}>0, \lim _{n \rightarrow \infty}\left\|x_{n+1}-x_{n}\right\|=0$ and $\alpha_{n} \rightarrow 0$, we obtain

$$
\lim _{n \rightarrow \infty}\left\|B_{2} x_{n}-B_{2} x^{*}\right\|=0
$$

and

$$
\lim _{n \rightarrow \infty}\left\|B_{1} v_{n}-B_{1} y^{*}\right\|=0 .
$$


Bnouhachem Fixed Point Theory and Applications 2014, 2014:235

Page 11 of 21

Since $T_{\mu_{2}}^{F_{2}}$ is firmly nonexpansive, we have

$$
\begin{aligned}
\left\|v_{n}-y^{*}\right\|^{2}= & \left\|T_{\mu_{2}}^{F_{2}}\left[x_{n}-\mu_{2} B_{2} x_{n}\right]-T_{\mu_{2}}^{F_{2}}\left[x^{*}-\mu_{2} B_{2} x^{*}\right]\right\|^{2} \\
\leq & \left\langle v_{n}-y^{*},\left(x_{n}-\mu_{2} B_{2} x_{n}\right)-\left(x^{*}-\mu_{2} B_{2} x^{*}\right)\right\rangle \\
= & \frac{1}{2}\left\{\left\|v_{n}-y^{*}\right\|^{2}+\left\|x_{n}-x^{*}-\mu_{2}\left(B_{2} x_{n}-B_{2} x^{*}\right)\right\|^{2}\right. \\
& \left.-\left\|x_{n}-x^{*}-\mu_{2}\left(B_{2} x_{n}-B_{2} x^{*}\right)-\left(v_{n}-y^{*}\right)\right\|^{2}\right\} \\
\leq & \frac{1}{2}\left\{\left\|v_{n}-y^{*}\right\|^{2}+\left\|x_{n}-x^{*}\right\|^{2}-\mu_{2}\left(2 \theta_{2}-\mu_{2}\right)\left\|B_{2} x_{n}-B_{2} x^{*}\right\|^{2}\right. \\
& \left.-\left\|x_{n}-x^{*}-\mu_{2}\left(B_{2} x_{n}-B_{2} x^{*}\right)-\left(v_{n}-y^{*}\right)\right\|^{2}\right\} \\
\leq & \frac{1}{2}\left\{\left\|v_{n}-y^{*}\right\|^{2}+\left\|x_{n}-x^{*}\right\|^{2}\right. \\
& \left.-\left\|x_{n}-v_{n}-\mu_{2}\left(B_{2} x_{n}-B_{2} x^{*}\right)-\left(x^{*}-y^{*}\right)\right\|^{2}\right\} \\
= & \frac{1}{2}\left\{\left\|v_{n}-y^{*}\right\|^{2}+\left\|x_{n}-x^{*}\right\|^{2}-\left\|x_{n}-v_{n}-\left(x^{*}-y^{*}\right)\right\|^{2}\right. \\
& \left.+2 \mu_{2}\left\langle x_{n}-v_{n}-\left(x^{*}-y^{*}\right), B_{2} x_{n}-B_{2} x^{*}\right\rangle-\mu_{2}^{2}\left\|B_{2} x_{n}-B_{2} x^{*}\right\|^{2}\right\} \\
\leq & \frac{1}{2}\left\{\left\|v_{n}-y^{*}\right\|^{2}+\left\|x_{n}-x^{*}\right\|^{2}-\left\|x_{n}-v_{n}-\left(x^{*}-y^{*}\right)\right\|^{2}\right. \\
& \left.+2 \mu_{2}\left\|x_{n}-v_{n}-\left(x^{*}-y^{*}\right)\right\|\left\|B_{2} x_{n}-B_{2} x^{*}\right\|\right\} .
\end{aligned}
$$

Hence, we get

$$
\begin{aligned}
\left\|v_{n}-y^{*}\right\|^{2} \leq & \left\|x_{n}-x^{*}\right\|^{2}-\left\|x_{n}-v_{n}-\left(x^{*}-y^{*}\right)\right\|^{2} \\
& +2 \mu_{2}\left\|x_{n}-v_{n}-\left(x^{*}-y^{*}\right)\right\|\left\|B_{2} x_{n}-B_{2} x^{*}\right\| .
\end{aligned}
$$

On the other hand, from (3.1) and Lemma 2.1(ii), we obtain

$$
\begin{aligned}
\left\|z_{n}-x^{*}\right\|^{2}= & \left\|T_{\mu_{1}}^{F_{1}}\left[v_{n}-\mu_{1} B_{1} v_{n}\right]-T_{\mu_{1}}^{F_{1}}\left[y^{*}-\mu_{1} B_{1} y^{*}\right]\right\|^{2} \\
\leq & \left\langle z_{n}-x^{*},\left(v_{n}-\mu_{1} B_{1} v_{n}\right)-\left(y^{*}-\mu_{1} B_{1} y^{*}\right)\right\rangle \\
= & \frac{1}{2}\left\{\left\|z_{n}-x^{*}\right\|^{2}+\left\|v_{n}-y^{*}-\mu_{1}\left(B_{1} v_{n}-B_{1} y^{*}\right)\right\|^{2}\right. \\
& \left.-\left\|v_{n}-y^{*}-\mu_{1}\left(B_{1} v_{n}-B_{1} y^{*}\right)-\left(z_{n}-x^{*}\right)\right\|^{2}\right\} \\
= & \frac{1}{2}\left\{\left\|z_{n}-x^{*}\right\|^{2}+\left\|v_{n}-y^{*}\right\|^{2}-2 \mu_{1}\left(v_{n}-y^{*}, B_{1} v_{n}-B_{1} y^{*}\right\rangle\right. \\
& \left.+\mu_{1}^{2}\left\|B_{1} v_{n}-B_{1} y^{*}\right\|^{2}-\left\|v_{n}-y^{*}-\mu_{1}\left(B_{1} v_{n}-B_{1} y^{*}\right)-\left(z_{n}-x^{*}\right)\right\|^{2}\right\} \\
\leq & \frac{1}{2}\left\{\left\|z_{n}-x^{*}\right\|^{2}+\left\|v_{n}-y^{*}\right\|^{2}-\mu_{1}\left(2 \theta_{1}-\mu_{1}\right)\left\|B_{1} v_{n}-B_{1} y^{*}\right\|^{2}\right. \\
& \left.-\left\|v_{n}-y^{*}-\mu_{1}\left(B_{1} v_{n}-B_{1} y^{*}\right)-\left(z_{n}-x^{*}\right)\right\|^{2}\right\} \\
\leq & \frac{1}{2}\left\{\left\|z_{n}-x^{*}\right\|^{2}+\left\|v_{n}-y^{*}\right\|^{2}\right. \\
& \left.-\left\|v_{n}-z_{n}-\mu_{1}\left(B_{1} v_{n}-B_{1} y^{*}\right)+\left(x^{*}-y^{*}\right)\right\|^{2}\right\}
\end{aligned}
$$




$$
\begin{aligned}
\leq & \frac{1}{2}\left\{\left\|z_{n}-x^{*}\right\|^{2}+\left\|v_{n}-y^{*}\right\|^{2}-\left\|v_{n}-z_{n}+\left(x^{*}-y^{*}\right)\right\|^{2}\right. \\
& \left.+2 \mu_{1}\left\langle v_{n}-z_{n}+\left(x^{*}-y^{*}\right), B_{1} v_{n}-B_{1} y^{*}\right)\right\} \\
\leq & \frac{1}{2}\left\{\left\|z_{n}-x^{*}\right\|^{2}+\left\|v_{n}-y^{*}\right\|^{2}-\left\|v_{n}-z_{n}+\left(x^{*}-y^{*}\right)\right\|^{2}\right. \\
& \left.+2 \mu_{1}\left\|v_{n}-z_{n}+\left(x^{*}-y^{*}\right)\right\|\left\|B_{1} v_{n}-B_{1} y^{*}\right\|\right\},
\end{aligned}
$$

which implies that

$$
\begin{aligned}
\left\|z_{n}-x^{*}\right\|^{2} \leq & \left\|v_{n}-y^{*}\right\|^{2}-\left\|v_{n}-z_{n}+\left(x^{*}-y^{*}\right)\right\|^{2} \\
& +2 \mu_{1}\left\|v_{n}-z_{n}+\left(x^{*}-y^{*}\right)\right\|\left\|B_{1} v_{n}-B_{1} y^{*}\right\| \\
\leq & \left\|x_{n}-x^{*}\right\|^{2}-\left\|x_{n}-v_{n}-\left(x^{*}-y^{*}\right)\right\|^{2} \\
& +2 \mu_{2}\left\|x_{n}-v_{n}-\left(x^{*}-y^{*}\right)\right\|\left\|B_{2} x_{n}-B_{2} x^{*}\right\| \\
& -\left\|v_{n}-z_{n}+\left(x^{*}-y^{*}\right)\right\|^{2}+2 \mu_{1}\left\|v_{n}-z_{n}+\left(x^{*}-y^{*}\right)\right\|\left\|B_{1} v_{n}-B_{1} y^{*}\right\|,
\end{aligned}
$$

where the last inequality follows from (3.10). From (3.9) and the above inequality, we have

$$
\begin{aligned}
\left\|x_{n+1}-x^{*}\right\|^{2} \leq & \gamma_{n}\left\|x_{n}-x^{*}\right\|^{2}+\left(1-\gamma_{n}\right)\left\|z_{n}-x^{*}\right\|^{2}+2 \alpha_{n}\left\langle\rho f\left(x_{n}\right)-\mu T\left(y_{n}\right), x_{n+1}\right\rangle \\
\leq & \gamma_{n}\left\|x_{n}-x^{*}\right\|^{2}+2 \alpha_{n}\left\langle\rho f\left(x_{n}\right)-\mu T\left(y_{n}\right), x_{n+1}\right\rangle+\left(1-\gamma_{n}\right)\left\|x_{n}-x^{*}\right\|^{2} \\
& +\left(1-\gamma_{n}\right)\left(-\left\|x_{n}-v_{n}-\left(x^{*}-y^{*}\right)\right\|^{2}\right. \\
& \left.+2 \mu_{2}\left\|x_{n}-v_{n}-\left(x^{*}-y^{*}\right)\right\|\left\|B_{2} x_{n}-B_{2} x^{*}\right\|\right) \\
& +\left(1-\gamma_{n}\right)\left(-\left\|v_{n}-z_{n}+\left(x^{*}-y^{*}\right)\right\|^{2}\right. \\
& \left.+2 \mu_{1}\left\|v_{n}-z_{n}+\left(x^{*}-y^{*}\right)\right\|\left\|B_{1} v_{n}-B_{1} y^{*}\right\|\right) \\
= & \left\|x_{n}-x^{*}\right\|^{2}+2 \alpha_{n}\left\langle\rho f\left(x_{n}\right)-\mu T\left(y_{n}\right), x_{n+1}\right\rangle \\
& +\left(1-\gamma_{n}\right)\left(-\left\|x_{n}-v_{n}-\left(x^{*}-y^{*}\right)\right\|^{2}\right. \\
& \left.+2 \mu_{2}\left\|x_{n}-v_{n}-\left(x^{*}-y^{*}\right)\right\|\left\|B_{2} x_{n}-B_{2} x^{*}\right\|\right) \\
& +\left(1-\gamma_{n}\right)\left(-\left\|v_{n}-z_{n}+\left(x^{*}-y^{*}\right)\right\|^{2}\right. \\
& \left.+2 \mu_{1}\left\|v_{n}-z_{n}+\left(x^{*}-y^{*}\right)\right\|\left\|B_{1} v_{n}-B_{1} y^{*}\right\|\right),
\end{aligned}
$$

which implies that

$$
\begin{aligned}
&\left(1-\gamma_{n}\right)\left(\left\|x_{n}-v_{n}-\left(x^{*}-y^{*}\right)\right\|^{2}+\left\|v_{n}-z_{n}+\left(x^{*}-y^{*}\right)\right\|^{2}\right) \\
& \leq 2 \alpha_{n}\left(\rho f\left(x_{n}\right)-\mu T\left(y_{n}\right), x_{n+1}\right\rangle+\left(\left\|x_{n}-x^{*}\right\|+\left\|x_{n+1}-x^{*}\right\|\right)\left\|x_{n+1}-x_{n}\right\| \\
&+2\left(1-\gamma_{n}\right) \mu_{2}\left\|x_{n}-v_{n}-\left(x^{*}-y^{*}\right)\right\|\left\|B_{2} x_{n}-B_{2} x^{*}\right\| \\
&+2\left(1-\gamma_{n}\right) \mu_{1}\left\|v_{n}-z_{n}+\left(x^{*}-y^{*}\right)\right\|\left\|B_{1} v_{n}-B_{1} y^{*}\right\| .
\end{aligned}
$$

Since $\lim _{n \rightarrow \infty}\left\|x_{n+1}-x_{n}\right\|=0, \lim _{n \rightarrow \infty} \alpha_{n} \rightarrow 0,0<\liminf \gamma_{n}<\lim \sup \gamma_{n}<1, \lim _{n \rightarrow \infty} \| B_{2} \times$ $x_{n}-B_{2} x^{*}\left\|=0, \lim _{n \rightarrow \infty}\right\| B_{1} v_{n}-B_{1} y^{*} \|=0$, we obtain

$$
\lim _{n \rightarrow \infty}\left\|x_{n}-v_{n}-\left(x^{*}-y^{*}\right)\right\|=0 \quad \text { and } \quad \lim _{n \rightarrow \infty}\left\|v_{n}-z_{n}+\left(x^{*}-y^{*}\right)\right\|=0
$$


Since

$$
\left\|x_{n}-z_{n}\right\| \leq\left\|x_{n}-v_{n}-\left(x^{*}-y^{*}\right)\right\|+\left\|v_{n}-z_{n}+\left(x^{*}-y^{*}\right)\right\|,
$$

we get

$$
\lim _{n \rightarrow \infty}\left\|x_{n}-z_{n}\right\|=0
$$

It follows from (3.8) and (3.11) that

$$
\lim _{n \rightarrow \infty}\left\|y_{n}-z_{n}\right\|=0
$$

We define a mapping $W: C \rightarrow H$ by $W x=\beta S x+(1-\beta) x$ with $\sigma \leq \beta<1$. It follows from Lemma 2.5 that $W$ is a nonexpansive mapping and $F(W)=F(S)$. Note that

$$
\begin{aligned}
\left\|W z_{n}-z_{n}\right\| & \leq\left\|W z_{n}-y_{n}\right\|+\left\|z_{n}-y_{n}\right\| \\
& \leq\left|\beta_{n}-\beta\right|\left\|S z_{n}-z_{n}\right\|+\left\|z_{n}-y_{n}\right\| .
\end{aligned}
$$

Since $\lim _{n \rightarrow \infty} \beta_{n}=\beta$ and $\lim _{n \rightarrow \infty}\left\|y_{n}-z_{n}\right\|=0$, we obtain

$$
\lim _{n \rightarrow \infty}\left\|W z_{n}-z_{n}\right\|=0
$$

Since $\left\{x_{n}\right\}$ is bounded and without loss of generality we can assume that $x_{n} \rightarrow x^{*} \in C$, from (3.11), it is easy to observe that $z_{n} \rightarrow x^{*}$. It follows from Lemma 2.3 that $x^{*} \in F(W)=F(S)$. Therefore $w_{w}\left(x_{n}\right) \subset F(S)$.

Theorem 3.1 The sequence $\left\{x_{n}\right\}$ generated by Algorithm 3.1 converges strongly to $z$, which is the unique solution of the variational inequality

$$
\langle\rho f(z)-\mu T(z), x-z\rangle \leq 0, \quad \forall x \in \Omega \cap F(S) .
$$

Proof Since $\left\{x_{n}\right\}$ is bounded $x_{n} \rightarrow w$ and from Lemma 3.2, we have $w \in F(S)$. Next, we show that $w \in \Omega$. Since $\lim _{n \rightarrow \infty}\left\|x_{n}-z_{n}\right\|=0$ and there exists a subsequence $\left\{x_{n_{k}}\right\}$ of $\left\{x_{n}\right\}$ such that $x_{n_{k}} \rightarrow w$, it is easy to observe that $z_{n_{k}} \rightarrow w$. For any $x, y \in C$, using (2.1), we have

$$
\begin{aligned}
\|Q(x)-Q(y)\|^{2}= & \| T_{\mu_{1}}^{F_{1}}\left[T_{\mu_{2}}^{F_{2}}\left[x-\mu_{2} B_{2} x\right]-\mu_{1} B_{1} T_{\mu_{2}}^{F_{2}}\left[x-\mu_{2} B_{2} x\right]\right] \\
& -T_{\mu_{1}}^{F_{1}}\left[T_{\mu_{2}}^{F_{2}}\left[y-\mu_{2} B_{2} y\right]-\mu_{1} B_{1} T_{\mu_{2}}^{F_{2}}\left[y-\mu_{2} B_{2} y\right]\right] \|^{2} \\
\leq & \|\left(T_{\mu_{2}}^{F_{2}}\left[x-\mu_{2} B_{2} x\right]-T_{\mu_{2}}^{F_{2}}\left[y-\mu_{2} B_{2} y\right]\right) \\
& -\mu_{1}\left(B_{1} T_{\mu_{2}}^{F_{2}}\left[x-\mu_{2} B_{2} x\right]-B_{1} T_{\mu_{2}}^{F_{2}}\left[y-\mu_{2} B_{2} y\right]\right) \|^{2} \\
\leq & \left\|T_{\mu_{2}}^{F_{2}}\left[x-\mu_{2} B_{2} x\right]-T_{\mu_{2}}^{F_{2}}\left[y-\mu_{2} B_{2} y\right]\right\|^{2} \\
& -\mu_{1}\left(2 \theta_{1}-\mu_{1}\right)\left\|B_{1} T_{\mu_{2}}^{F_{2}}\left[x-\mu_{2} B_{2} x\right]-B_{1} T_{\mu_{2}}^{F_{2}}\left[y-\mu_{2} B_{2} y\right]\right\|^{2} \\
\leq & \left\|T_{\mu_{2}}^{F_{2}}\left[x-\mu_{2} B_{2} x\right]-T_{\mu_{2}}^{F_{2}}\left[y-\mu_{2} B_{2} y\right]\right\|^{2} \\
\leq & \left\|\left(x-\mu_{2} B_{2} x\right)-\left(y-\mu_{2} B_{2} y\right)\right\|^{2}
\end{aligned}
$$




$$
\begin{aligned}
& \leq\|x-y\|^{2}-\mu_{2}\left(2 \theta_{2}-\mu_{2}\right)\left\|B_{2} x-B_{2} y\right\|^{2} \\
& \leq\|x-y\|^{2} .
\end{aligned}
$$

This implies that $Q: C \rightarrow C$ is nonexpansive. On the other hand

$$
\begin{aligned}
\left\|z_{n}-Q\left(z_{n}\right)\right\|^{2} & =\left\|T_{\mu_{1}}^{F_{1}}\left[T_{\mu_{2}}^{F_{2}}\left[x_{n}-\mu_{2} B_{2} x_{n}\right]-\mu_{1} B_{1} T_{\mu_{2}}^{F_{2}}\left[x_{n}-\mu_{2} B_{2} x_{n}\right]\right]-Q\left(z_{n}\right)\right\|^{2} \\
& =\left\|Q\left(x_{n}\right)-Q\left(z_{n}\right)\right\|^{2} \\
& \leq\left\|x_{n}-z_{n}\right\|^{2} .
\end{aligned}
$$

Since $\lim _{n \rightarrow \infty}\left\|x_{n}-z_{n}\right\|=0$ (see (3.11)), we have $\lim _{n \rightarrow \infty}\left\|z_{n}-Q\left(z_{n}\right)\right\|=0$. It follows from Lemma 2.3 that $w=Q(w)$, which implies from Lemma 2.2 that $w \in \Omega$. Thus we have

$$
w \in \Omega \cap F(S) .
$$

Since $0 \leq \rho \tau<\mu \eta$, from Lemma 2.4 , the operator $\mu T-\rho f$ is $\mu \eta$ - $\rho \tau$-strongly monotone, and we get the uniqueness of the solution of the variational inequality (3.13) and denote it by $z \in \Omega \cap F(S)$.

Next, we claim that $\lim _{\sup _{n \rightarrow \infty}}\left\langle\rho f(z)-\mu T(z), x_{n}-z\right\rangle \leq 0$. Since $\left\{x_{n}\right\}$ is bounded, there exists a subsequence $\left\{x_{n_{k}}\right\}$ of $\left\{x_{n}\right\}$ such that

$$
\begin{aligned}
& \limsup _{n \rightarrow \infty}\left\langle\rho f(z)-\mu T(z), x_{n}-z\right\rangle \\
& \quad=\limsup _{k \rightarrow \infty}\left\langle\rho f(z)-\mu T(z), x_{n_{k}}-z\right\rangle \\
& =\langle\rho f(z)-\mu T(z), w-z\rangle \leq 0 .
\end{aligned}
$$

Next, we show that $x_{n} \rightarrow z$. We have

$$
\begin{aligned}
\left\|x_{n+1}-z\right\|^{2}= & \left\langle\alpha_{n} \rho f\left(x_{n}\right)+\gamma_{n} x_{n}+\left(\left(1-\gamma_{n}\right) I-\alpha_{n} \mu T\right)\left(y_{n}\right)-z, x_{n+1}-z\right\rangle \\
= & \alpha_{n}\left\langle\rho f\left(x_{n}\right)-\mu T(z), x_{n+1}-z\right\rangle+\gamma_{n}\left\langle x_{n}-z, x_{n+1}-z\right\rangle \\
& +\left\langle\left(\left(1-\gamma_{n}\right) I-\alpha_{n} \mu T\right)\left(y_{n}\right)-\left(\left(1-\gamma_{n}\right) I-\alpha_{n} \mu T\right)(z), x_{n+1}-z\right\rangle \\
\leq & \alpha_{n}\left\langle\rho\left(f\left(x_{n}\right)-f(z)\right), x_{n+1}-z\right\rangle+\alpha_{n}\left\langle\rho f(z)-\mu T(z), x_{n+1}-z\right\rangle \\
& +\gamma_{n}\left\|x_{n}-z\right\|\left\|x_{n+1}-z\right\|+\left(1-\gamma_{n}-\alpha_{n} v\right)\left\|y_{n}-z\right\|\left\|x_{n+1}-z\right\| \\
\leq & \alpha_{n} \rho \tau\left\|x_{n}-z\right\|\left\|x_{n+1}-z\right\|+\alpha_{n}\left\langle\rho f(z)-\mu T(z), x_{n+1}-z\right\rangle \\
& +\gamma_{n}\left\|x_{n}-z\right\|\left\|x_{n+1}-z\right\|+\left(1-\gamma_{n}-\alpha_{n} v\right)\left\|x_{n}-z\right\|\left\|x_{n+1}-z\right\| \\
= & \left(1-\alpha_{n}(v-\rho \tau)\right)\left\|x_{n}-z\right\|\left\|x_{n+1}-z\right\|+\alpha_{n}\left\langle\rho f(z)-\mu T(z), x_{n+1}-z\right\rangle \\
\leq & \frac{1-\alpha_{n}(v-\rho \tau)}{2}\left(\left\|x_{n}-z\right\|^{2}+\left\|x_{n+1}-z\right\|^{2}\right) \\
& +\alpha_{n}\left\langle\rho f(z)-\mu T(z), x_{n+1}-z\right\rangle \\
\leq & \frac{1-\alpha_{n}(v-\rho \tau)}{2}\left\|x_{n}-z\right\|^{2}+\frac{1}{2}\left\|x_{n+1}-z\right\|^{2} \\
& +\alpha_{n}\left\langle\rho f(z)-\mu T(z), x_{n+1}-z\right\rangle,
\end{aligned}
$$


which implies that

$$
\left\|x_{n+1}-z\right\|^{2} \leq\left(1-\alpha_{n}(v-\rho \tau)\right)\left\|x_{n}-z\right\|^{2}+2 \alpha_{n}\left\langle\rho f(z)-\mu T(z), x_{n+1}-z\right\rangle
$$

Let $v_{n}=\alpha_{n}(v-\rho \tau)$ and $\delta_{n}=2 \alpha_{n}\left\langle\rho f(z)-\mu T(z), x_{n+1}-z\right\rangle$.

We have

$$
\sum_{n=1}^{\infty} \alpha_{n}=\infty
$$

and

$$
\limsup _{n \rightarrow \infty}\left\{\frac{1}{v-\rho \tau}\left\langle\rho f(z)-\mu T(z), x_{n+1}-z\right\rangle\right\} \leq 0
$$

It follows that

$$
\sum_{n=1}^{\infty} v_{n}=\infty \quad \text { and } \quad \limsup _{n \rightarrow \infty} \frac{\delta_{n}}{v_{n}} \leq 0
$$

Thus all the conditions of Lemma 2.8 are satisfied. Hence we deduce that $x_{n} \rightarrow z$. This completes the proof.

\section{Applications}

To verify the theoretical assertions, we consider the following example.

Example 4.1 Let $\alpha_{n}=\frac{1}{3 n}, \beta_{n}=\frac{1}{n^{3}}$, and $\gamma_{n}=\frac{2 n-1}{3 n}$.

It is easy to show that the sequence $\left\{\gamma_{n}\right\}$ satisfies condition (a).

We have

$$
\lim _{n \rightarrow \infty} \alpha_{n}=\frac{1}{3} \lim _{n \rightarrow \infty} \frac{1}{n}=0
$$

and

$$
\sum_{n=1}^{\infty} \alpha_{n}=\frac{1}{3} \sum_{n=1}^{\infty} \frac{1}{n}=\infty
$$

The sequence $\left\{\alpha_{n}\right\}$ satisfies condition (b).

Let $\mathbb{R}$ be the set of real numbers, $B_{1}=B_{2}=0$, and let the mapping $T: \mathbb{R} \rightarrow \mathbb{R}$ be defined by

$$
T(x)=\frac{2 x+5}{7}, \quad \forall x \in \mathbb{R}
$$

let the mapping $S: \mathbb{R} \rightarrow \mathbb{R}$ be defined by

$$
S(x)=\frac{x}{3}, \quad \forall x \in \mathbb{R}
$$


let the mapping $f: \mathbb{R} \rightarrow \mathbb{R}$ be defined by

$$
f(x)=\frac{x}{14}, \quad \forall x \in \mathbb{R}
$$

It is easy to show that $T$ is a 1-Lipschitzian mapping and $\frac{1}{7}$-strongly monotone, $S$ is a 0 -strict pseudo-contraction mapping and $f$ is $\frac{1}{7}$-Lipschitzian. Let the mapping $F_{2}: \mathbb{R} \times$ $\mathbb{R} \rightarrow \mathbb{R}$ be defined by

$$
F_{2}(x, y)=-5 x^{2}+x y+4 y^{2}, \quad \forall(x, y) \in \mathbb{R} \times \mathbb{R} .
$$

By the definition of $F_{2}$, we have

$$
\begin{aligned}
0 & \leq F_{2}\left(u_{n}, y\right)+\frac{1}{\mu_{2}}\left\langle y-u_{n}, u_{n}-x_{n}\right\rangle \\
& =-5 u_{n}^{2}+u_{n} y+4 y^{2}+\frac{1}{\mu_{2}}\left(y-u_{n}\right)\left(u_{n}-x_{n}\right) .
\end{aligned}
$$

Then

$$
\begin{aligned}
0 & \leq \mu_{2}\left(-5 u_{n}^{2}+u_{n} y+4 y^{2}\right)+\left(y u_{n}-y x_{n}-u_{n}^{2}+u_{n} x_{n}\right) \\
& =4 \mu_{2} y^{2}+\left(\mu_{2} u_{n}+u_{n}-x_{n}\right) y-5 \mu_{2} u_{n}^{2}-u_{n}^{2}+u_{n} x_{n} .
\end{aligned}
$$

Let $A(y)=4 \mu_{2} y^{2}+\left(\mu_{2} u_{n}+u_{n}-x_{n}\right) y-5 \mu_{2} u_{n}^{2}-u_{n}^{2}+u_{n} x_{n} . A(y)$ is a quadratic function of $y$ with coefficients $a=4 \mu_{2}, b=\mu_{2} u_{n}+u_{n}-x_{n}, c=-5 \mu_{2} u_{n}^{2}-u_{n}^{2}+u_{n} x_{n}$. We determine the discriminant $\Delta$ of $A$ as follows:

$$
\begin{aligned}
\Delta & =b^{2}-4 a c \\
& =\left(\mu_{2} u_{n}+u_{n}-x_{n}\right)^{2}-16 \mu_{2}\left(-5 \mu_{2} u_{n}^{2}-u_{n}^{2}+u_{n} x_{n}\right) \\
& =81 \mu_{2}^{2} u_{n}^{2}+18 \mu_{2} u_{n}^{2}+u_{n}^{2}-18 \mu_{2} u_{n} x_{n}-2 u_{n} x_{n}+x_{n}^{2} \\
& =x_{n}^{2}+\left(81 \mu_{2}^{2}+18 \mu_{2}+1\right) u_{n}^{2}-2 x_{n} u_{n}\left(9 \mu_{2}+1\right) \\
& =\left(x_{n}-u_{n}\left(9 \mu_{2}+1\right)\right)^{2} .
\end{aligned}
$$

We have $A(y) \geq 0, \forall y \in \mathbb{R}$. If it has at most one solution in $\mathbb{R}$, then $\Delta=0$, we obtain

$$
u_{n}=\frac{x_{n}}{1+9 \mu_{2}} \text {. }
$$

Let the mapping $F_{1}: \mathbb{R} \times \mathbb{R} \rightarrow \mathbb{R}$ be defined by

$$
F_{1}(x, y)=-3 x^{2}+x y+2 y^{2}, \quad \forall(x, y) \in \mathbb{R} \times \mathbb{R} .
$$

By the definition of $F_{1}$, we have

$$
\begin{aligned}
0 & \leq F_{1}\left(u_{n}, y\right)+\frac{1}{\mu_{1}}\left\langle y-u_{n}, u_{n}-x_{n}\right\rangle \\
& =-3 u_{n}^{2}+u_{n} y+2 y^{2}+\frac{1}{\mu_{1}}\left(y-u_{n}\right)\left(u_{n}-x_{n}\right) .
\end{aligned}
$$


Then

$$
\begin{aligned}
0 & \leq \mu_{1}\left(-3 u_{n}^{2}+u_{n} y+2 y^{2}\right)+\left(y u_{n}-y x_{n}-u_{n}^{2}+u_{n} x_{n}\right) \\
& =2 \mu_{1} y^{2}+\left(\mu_{1} u_{n}+u_{n}-x_{n}\right) y-3 \mu_{1} u_{n}^{2}-u_{n}^{2}+u_{n} x_{n} .
\end{aligned}
$$

Let $B(y)=2 \mu_{1} y^{2}+\left(\mu_{1} u_{n}+u_{n}-x_{n}\right) y-3 \mu_{1} u_{n}^{2}-u_{n}^{2}+u_{n} x_{n}$. $B(y)$ is a quadratic function of $y$ with coefficients $a=2 \mu_{1}, b=\mu_{1} u_{n}+u_{n}-x_{n}, c=-3 \mu_{1} u_{n}^{2}-u_{n}^{2}+u_{n} x_{n}$. We determine the discriminant $\Delta$ of $B$ as follows:

$$
\begin{aligned}
\Delta & =b^{2}-4 a c \\
& =\left(\mu_{1} u_{n}+u_{n}-x_{n}\right)^{2}-8 \mu_{1}\left(-3 \mu_{1} u_{n}^{2}-u_{n}^{2}+u_{n} x_{n}\right) \\
& =u_{n}^{2}+10 \mu_{1} u_{n}^{2}+25 u_{n}^{2} \mu_{1}^{2}-2 x_{n} u_{n}-10 x_{n} u_{n} \mu_{1}+x_{n}^{2} \\
& =\left(u_{n}+5 u_{n} \mu_{1}\right)^{2}-2 x_{n}\left(u_{n}+5 u_{n} \mu_{1}\right)+x_{n}^{2} \\
& =\left(u_{n}+5 u_{n} \mu_{1}-x_{n}\right)^{2} .
\end{aligned}
$$

We have $B(y) \geq 0, \forall y \in \mathbb{R}$. If it has at most one solution in $\mathbb{R}$, then $\Delta=0$, we obtain

$$
u_{n}=\frac{x_{n}}{1+5 \mu_{1}} .
$$

For every $n \geq 1$, from (4.1) and (4.2), we rewrite (3.1) as follows:

$$
\left\{\begin{array}{l}
z_{n}=\frac{x_{n}}{\left(1+5 \mu_{1}\right)\left(1+9 \mu_{2}\right)} ; \\
y_{n}=\frac{z_{n}}{3 n^{3}}+\left(1-\frac{1}{n^{3}}\right) z_{n} ; \\
x_{n+1}=\left(\frac{\rho}{42 n}+\frac{2 n-1}{3 n}\right) x_{n}+\frac{(n+1) y_{n}}{3 n}-\mu \frac{2 y_{n}+5}{21 n} .
\end{array}\right.
$$

\begin{tabular}{|c|c|c|c|c|c|c|c|c|c|}
\hline & \multicolumn{3}{|c|}{ Algorithm 3.1} & \multicolumn{3}{|c|}{ Algorithm 3.2 with $F=F_{1}$} & \multicolumn{3}{|c|}{ Algorithm 3.2 with $F=F_{2}$} \\
\hline & $z_{n}$ & $y_{n}$ & $x_{n}$ & $z_{n}$ & $y_{n}$ & $x_{n}$ & $z_{n}$ & $y_{n}$ & $x_{n}$ \\
\hline$n=1$ & 0.714286 & 0.238095 & 10.000000 & 2.857143 & 0.952381 & 10.000000 & 2.500000 & 0.833333 & 10.000000 \\
\hline$n=2$ & 0.174115 & 0.159605 & 3.470684 & 0.908574 & 0.832860 & 3.937156 & 0.839002 & 0.769085 & 3.859410 \\
\hline$n=3$ & 0.078010 & 0.076084 & 1.799806 & 0.497992 & 0.485696 & 2.365460 & 0.472515 & 0.460848 & 2.295072 \\
\hline$n=4$ & 0.040919 & 0.040493 & 1.022977 & 0.303544 & 0.300382 & 1.517720 & 0.293530 & 0.290472 & 1.467648 \\
\hline$n=5$ & 0.023015 & 0.022893 & 0.605373 & 0.193853 & 0.192819 & 1.001573 & 0.190192 & 0.189177 & 0.968249 \\
\hline$n=6$ & 0.013425 & 0.013383 & 0.365708 & 0.126958 & 0.126566 & 0.671062 & 0.126044 & 0.125655 & 0.649610 \\
\hline$n=7$ & 0.007982 & 0.007966 & 0.223090 & 0.084379 & 0.084215 & 0.453535 & 0.084628 & 0.084464 & 0.440068 \\
\hline$n=8$ & 0.004782 & 0.004776 & 0.136314 & 0.056557 & 0.056483 & 0.307922 & 0.057242 & 0.057167 & 0.299676 \\
\hline$n=9$ & 0.002859 & 0.002856 & 0.082755 & 0.038063 & 0.038028 & 0.209346 & 0.038845 & 0.038809 & 0.204446 \\
\hline$n=10$ & 0.001685 & 0.001684 & 0.049394 & 0.025624 & 0.025607 & 0.142095 & 0.026353 & 0.026336 & 0.139297 \\
\hline
\end{tabular}

In all tests we take $\rho=\frac{1}{15}$ and $\mu=\frac{1}{7}$. In our example $\eta=\frac{1}{7}, k=1, \tau=\frac{1}{7}$. It is easy to show that the parameters satisfy $0<\mu<\frac{2 \eta}{k^{2}}, 0 \leq \rho \tau<\nu$, where $v=\mu\left(\eta-\frac{\mu k^{2}}{2}\right)$. All codes were written in Matlab, the values of $\left\{z_{n}\right\},\left\{y_{n}\right\}$, and $\left\{x_{n}\right\}$ with different $n$ are reported in Tables 1 and 2 .

Remark 4.1 Tables 1 and 2, and Figures 1 and 2 show that the sequences $\left\{z_{n}\right\},\left\{y_{n}\right\}$, and $\left\{x_{n}\right\}$ converge to 0 , where $\{0\}=\Omega \cap F(S)$. Also Tables 1 and 2 show that the convergence of Algorithm 3.1 is faster than Algorithm 3.2.

Table 1 The values of $\left\{z_{n}\right\},\left\{y_{n}\right\}$, and $\left\{x_{n}\right\}$ with initial value $x_{1}=10$ 
Table 2 The values of $\left\{z_{n}\right\},\left\{y_{n}\right\}$, and $\left\{x_{n}\right\}$ with initial value $x_{1}=-10$

\begin{tabular}{|c|c|c|c|c|c|c|c|c|c|}
\hline & \multicolumn{3}{|c|}{ Algorithm 3.1} & \multicolumn{3}{|c|}{ Algorithm 3.2 with $F=F_{1}$} & \multicolumn{3}{|c|}{ Algorithm 3.2 with $F=F_{2}$} \\
\hline & $z_{n}$ & $y_{n}$ & $x_{n}$ & $z_{n}$ & $y_{n}$ & $x_{n}$ & $z_{n}$ & $y_{n}$ & $x_{n}$ \\
\hline$=1$ & -0.714286 & -0.238095 & -10.000000 & -2.857143 & -0.952381 & -10.000000 & -2.500000 & -0.833333 & -10.000000 \\
\hline & 27 & & & $-c$ & & & & & \\
\hline & -0.0 & & 0 & & & & & & \\
\hline$=4$ & -0. & & & & & & & & \\
\hline$=5$ & -0.0 & -0.0 & & & & & & -0 & \\
\hline$n=6$ & -0.015158 & -0.015111 & -0.412926 & -0.1377 & -0.137358 & -0.728280 & & & -0.706715 \\
\hline $7=7$ & -0.009444 & -0.009426 & -0.263964 & -0.093772 & -0.093590 & -0.504027 & -0.094344 & -0.094161 & -0.490588 \\
\hline & -0.006031 & -0.006023 & -0.171898 & -0.064738 & -0.064654 & -0.352462 & -0.065776 & -0.065690 & -0.344356 \\
\hline$n=9$ & -0.00393 & -0.0039 & & -0.0452 & -0.045185 & -0.2487 & -0.046372 & -0.046330 & -0.244064 \\
\hline$n=10$ & -0.002627 & -0.002626 & -0.077009 & -0.031937 & -0.031916 & -0.177107 & -0.033029 & -0.033007 & -0.174582 \\
\hline
\end{tabular}
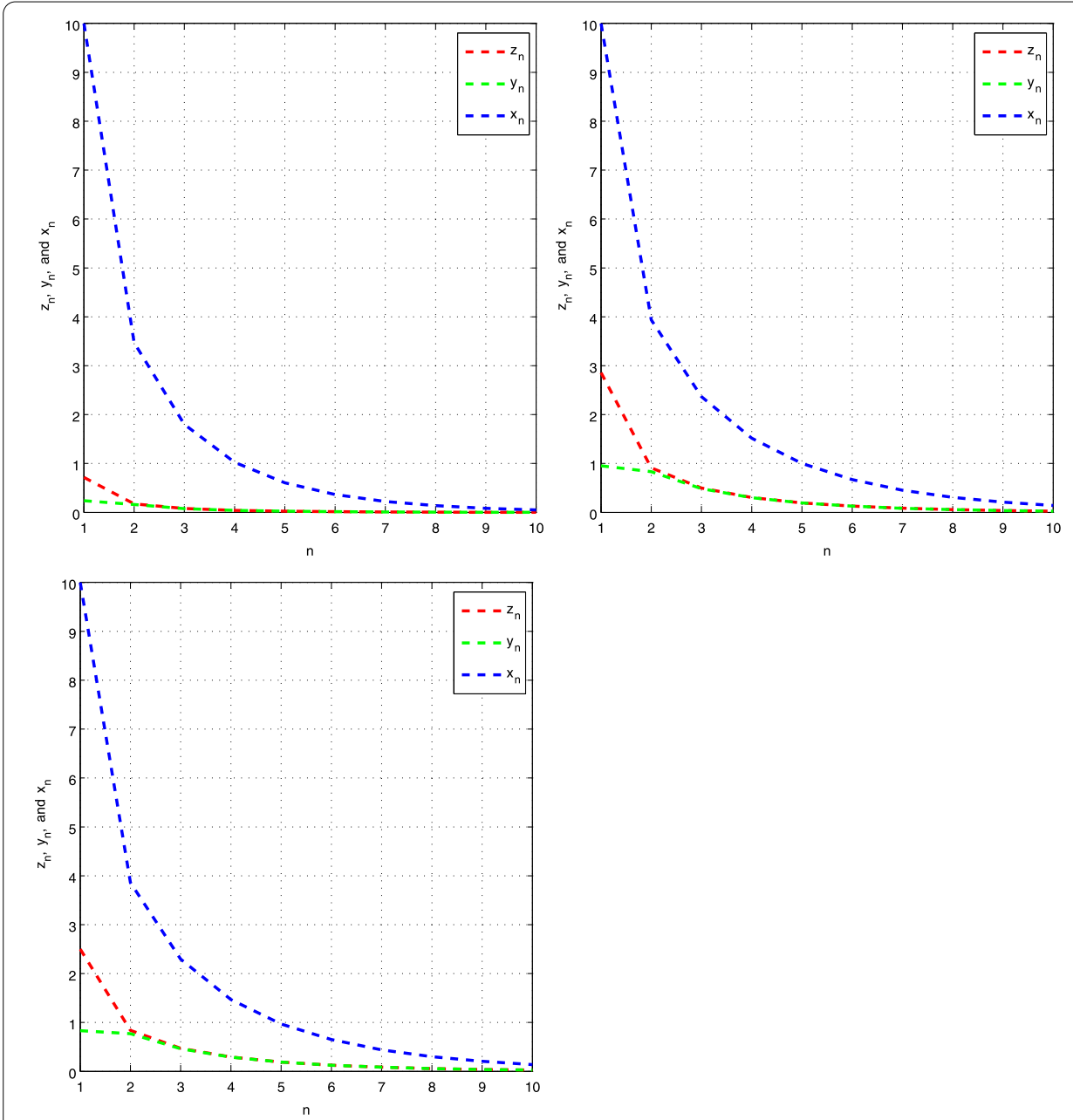

Figure 1 The convergence of $\left\{z_{n}\right\},\left\{y_{n}\right\}$, and $\left\{x_{n}\right\}$ with initial value $x_{1}=10$ for Algorithm 3.1 and Algorithm 3.2 with $F=F_{1}$ and with $F=F_{2}$. 

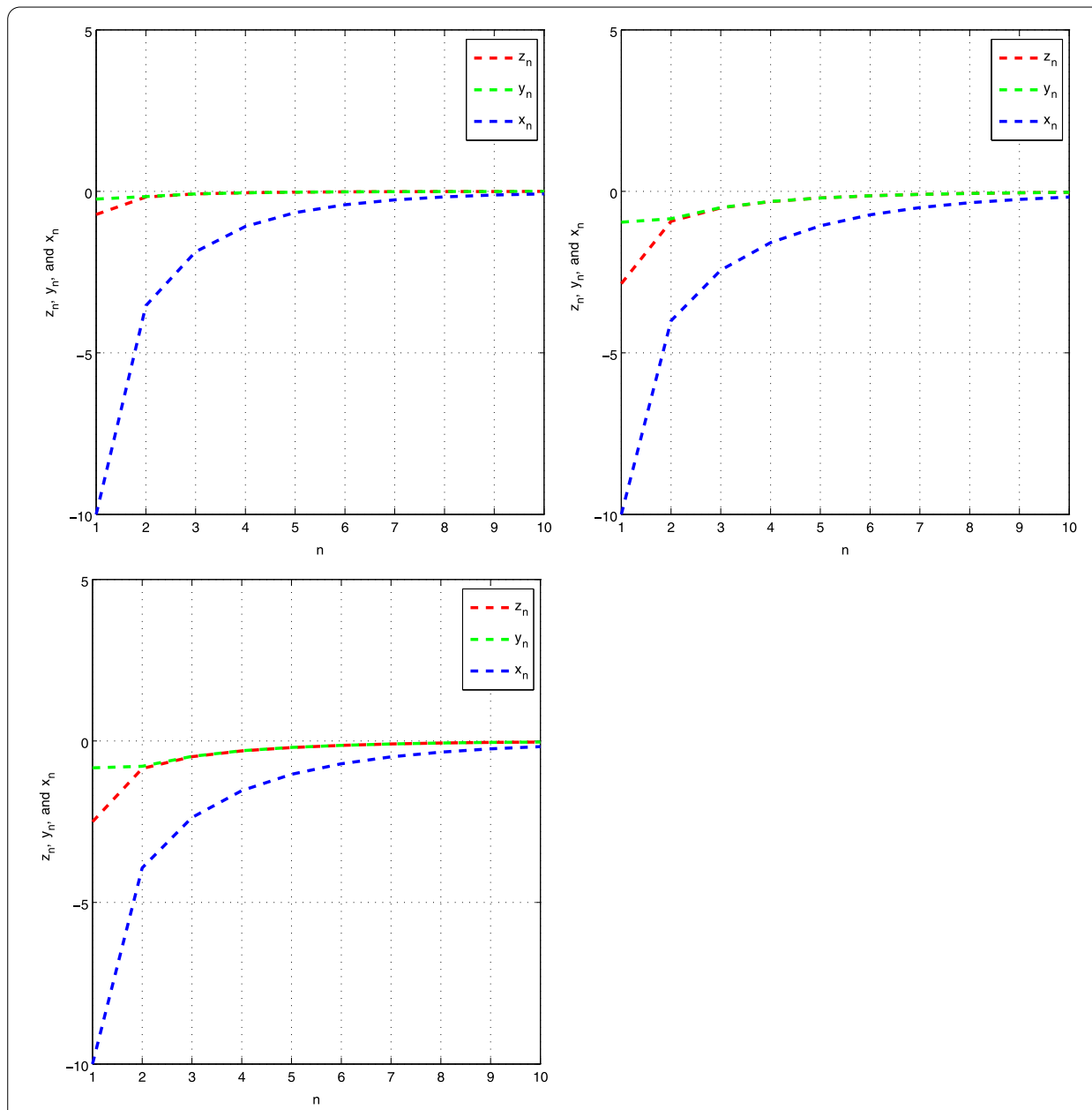

Figure 2 The convergence of $\left\{z_{n}\right\},\left\{y_{n}\right\}$, and $\left\{x_{n}\right\}$ with initial value $x_{1}=-10$ for Algorithm 3.1 and Algorithm 3.2 with $F=F_{1}$ and with $F=F_{2}$.

\section{Conclusions}

In this paper, we suggest and analyze an iterative method for finding the approximate element of the common set of solutions of (1.1) and (1.6) in real Hilbert space, which can be viewed as a refinement and improvement of some existing methods for solving equilibrium problem, and a hierarchical fixed point problem. Strong convergence of the proposed method is proved under mild assumptions. Furthermore, some preliminary numerical results are reported to verify the theoretical assertions of the proposed method and show that our algorithm for the system of generalized equilibrium problems is more attractive in practice than our algorithm for the generalized equilibrium problems.

Competing interests

The author declares that he has no competing interests.

\section{Acknowledgements}

The author would like to thank Prof. Xindan Li, Dean of School of Management and Engineering of Nanjing University, for providing excellent research facilities. 


\section{References}

1. Ceng, LC, Yao, JC: A relaxed extragradient-like method for a generalized mixed equilibrium problem, a general system of generalized equilibria and a fixed point problem. Nonlinear Anal. 72, 1922-1937 (2010)

2. Takahashi, S, Takahashi, W: Strong convergence theorem for a generalized equilibrium problem and a nonexpansive mapping in a Hilbert space. Nonlinear Anal. 69, 1025-1033 (2008)

3. Ceng, LC, Wang, CY, Yao, JC: Strong convergence theorems by a relaxed extragradient method for a general system of variational inequalities. Math. Methods Oper. Res. 67, 375-390 (2008)

4. Ceng, LC, Ansari, QH, Schaible, S, Yao, JC: Iterative methods for generalized equilibrium problems, systems of general generalized equilibrium problems and fixed point problems for nonexpansive mappings in Hilbert spaces. Fixed Point Theory 12(2), 293-308 (2011)

5. Ansari, QH: Metric Spaces: Including Fixed Point Theory and Set-Valued Maps. Narosa Publishing House, New Delhi (2010)

6. Reich, S, Sabach, S: Three strong convergence theorems regarding iterative methods for solving equilibrium problems in reflexive Banach spaces. Contemp. Math. 568, 22-240 (2012)

7. Latif, A, Al-Mazrooei, AE, Alofi, AS, Yao, JC: Hybrid iterative method for systems of generalized equilibria with constraints of variational inclusion and fixed point problems. Fixed Point Theory Appl. 2014, 164 (2014)

8. Ceng, LC, Al-Mezel, SA, Anasri, QH: Implicit and explicit iterative methods for systems of variational inequalities and zeros of accretive operators. Abstr. Appl. Anal. 2013, Article ID 631382 (2013)

9. Ansari, QH, Yao, JC: Systems of generalized variational inequalities and their applications. Appl. Anal. 76, 203-217 (2000)

10. Aubin, JP: Mathematical Methods of Game and Economic Theory. North-Holland, Amsterdam (1979)

11. Facchinei, F, Pang, JS: Finite-Dimensional Variational Inequalities and Complementarity Problems, vol. II. Springer, New York (2003)

12. Verma, RU: Projection methods, algorithms, and a new system of nonlinear variational inequalities. Comput. Math Appl. 41, 1025-1031 (2001)

13. Verma, RU: General convergence analysis for two-step projection methods and applications to variational problems. Appl. Math. Lett. 18, 1286-1292 (2005)

14. Ansari, QH, Wong, NC, Yao, JC: The existence of nonlinear inequalities. Appl. Math. Lett. 12(5), 89-92 (1999)

15. Bnouhachem, A: A self-adaptive method for solving general mixed variational inequalities. J. Math. Anal. Appl. 309(1), 136-150 (2005)

16. Bnouhachem, A: A new projection and contraction method for linear variational inequalities. J. Math. Anal. Appl. 314(2), 513-525 (2006)

17. Ansari, QH, Lalitha, CS, Mehta, M: Generalized Convexity, Nonsmooth Variational Inequalities and Nonsmooth Optimization. CRC Press, Boca Raton (2014)

18. Ansari, QH, Rehan, A: Split feasibility and fixed point problems. In: Ansari, QH (ed.) Nonlinear Analysis: Approximation Theory, Optimization and Applications, pp. 281-322. Birkhäuser, Basel (2014)

19. Zhou, $\mathrm{H}$ : Convergence theorems of fixed points for $\mathrm{k}$-strict pseudo-contractions in Hilbert spaces. Nonlinear Anal. 69 456-462 (2008)

20. Yao, Y, Cho, YJ, Liou, YC: Iterative algorithms for hierarchical fixed points problems and variational inequalities. Math. Comput. Model. 52(9-10), 1697-1705 (2010)

21. Crombez, G: A hierarchical presentation of operators with fixed points on Hilbert spaces. Numer. Funct. Anal. Optim 27, 259-277 (2006)

22. Mainge, PE, Moudafi, A: Strong convergence of an iterative method for hierarchical fixed-point problems. Pac. J. Optim. 3(3), 529-538 (2007)

23. Moudafi, A: Krasnoselski-Mann iteration for hierarchical fixed-point problems. Inverse Problems 23(4), 1635-1640 (2007)

24. Cianciaruso, F, Marino, G, Muglia, L, Yao, Y: On a two-steps algorithm for hierarchical fixed point problems and variational inequalities. J. Inequal. Appl. 2009, Article ID 208692 (2009)

25. Gu, G, Wang, S, Cho, YJ: Strong convergence algorithms for hierarchical fixed points problems and variational inequalities. J. Appl. Math. 2011, Article ID 164978 (2011)

26. Marino, G, Xu, HK: Explicit hierarchical fixed point approach to variational inequalities. J. Optim. Theory Appl. 149(1), $61-78(2011)$

27. Ceng, LC, Ansari, QH, Yao, JC: Iterative methods for triple hierarchical variational inequalities in Hilbert spaces. J. Optim. Theory Appl. 151, 489-512 (2011)

28. Bnouhachem, A, Noor, MA: An iterative method for approximating the common solutions of a variational inequality, a mixed equilibrium problem and a hierarchical fixed point problem. J. Inequal. Appl. 2013, 490 (2013)

29. Bnouhachem, A: Algorithms of common solutions for a variational inequality, a split equilibrium problem and a hierarchical fixed point problem. Fixed Point Theory Appl. 2013, 278 (2013)

30. Bnouhachem, A: An iterative method for a common solution of generalized mixed equilibrium problems, variational inequalities, and hierarchical fixed point problems. Fixed Point Theory Appl. 2014, 155 (2014)

31. Bnouhachem, $A$ : A hybrid iterative method for a combination of equilibrium problem, a combination of variational inequality problem and a hierarchical fixed point problem. Fixed Point Theory Appl. 2014, 163 (2014)

32. Bnouhachem, A, Al-Homidan, S, Ansari, QH: An iterative method for common solutions of equilibrium problems and hierarchical fixed point problems. Fixed Point Theory Appl. 2014, 194 (2014)

33. Bnouhachem, A: A modified projection method for a common solution of a system of variational inequalities, a split equilibrium problem and a hierarchical fixed-point problem. Fixed Point Theory Appl. 2014, 22 (2014)

34. Bnouhachem, A: Strong convergence algorithm for approximating the common solutions of a variational inequality, a mixed equilibrium problem and a hierarchical fixed-point problem. J. Inequal. Appl. 2014, 154 (2014)

35. Ceng, LC, Al-Mezel, SA, Latif, A: Hybrid viscosity approaches to general systems of variational inequalities with hierarchical fixed point problem constraints in Banach spaces. Abstr. Appl. Anal. 2014, Article ID 945985 (2014)

36. Ceng, LC, Khan, AR, Ansari, OH, Yao, JC: Viscosity approximation methods for strongly positive and monotone operators. Fixed Point Theory 10(1), 35-71 (2009) 
37. Ceng, LC, Anasri, QH, Yao, JC: Some iterative methods for finding fixed points and for solving constrained convex minimization problems. Nonlinear Anal. 74(16), 5286-5302 (2011)

38. Wang, $Y, X u, W$ : Strong convergence of a modified iterative algorithm for hierarchical fixed point problems and variational inequalities. Fixed Point Theory Appl. 2013, 121 (2013)

39. Ansari, QH, Ceng, LC, Gupta, H: Triple hierarchical variational inequalities. In: Ansari, QH (ed.) Nonlinear Analysis: Approximation Theory, Optimization and Applications, pp. 231-280. Birkhäuser, Basel (2014)

40. Censor, Y, Gibali, A, Reich, S: Algorithms for the split variational inequality problem. Numer. Algorithms 59(2), 301-323 (2012)

41. Moudafi, A: Split monotone variational inclusions. J. Optim. Theory Appl. 50, 275-283 (2011)

42. Blum, E, Oettli, W: From optimization and variational inequalities to equilibrium problems. Math. Stud. 63, 123-145 (1994)

43. Combettes, PL, Hirstoaga, SA: Equilibrium programming using proximal-like algorithms. Math. Program. 78, 29-41 (1997)

44. Geobel, K, Kirk, WA: Topics in Metric Fixed Point Theory. Stud. Adv. Math., vol. 28. Cambridge University Press, Cambridge (1990)

45. Zhou, HY: Convergence theorems of fixed points for $\kappa$-strict pseudo-contractions in Hilbert spaces. Nonlinear Anal. 69, 456-462 (2008)

46. Deng, BC, Chen, T, Li, ZF: Cyclic iterative method for strictly pseudononspreading in Hilbert space. J. Appl. Math. 2012, Article ID 435676 (2012)

47. Suzuki, T: Strong convergence of Krasnoselskii and Mann's type sequences for one parameter nonexpansive semigroups without Bochner integrals. J. Math. Anal. Appl. 305(1), 227-239 (2005)

48. Xu, HK: Iterative algorithms for nonlinear operators. J. Lond. Math. Soc. 66, 240-256 (2002)

49. Acedo, GL, Xu, HK: Iterative methods for strict pseudo-contractions in Hilbert space. Nonlinear Anal. 67(7), 2258-2271 (2007)

50. Marino, G, Xu, HK: Convergence of generalized proximal point algorithms. Commun. Pure Appl. Anal. 3, 791-808 (2004)

10.1186/1687-1812-2014-235

Cite this article as: Bnouhachem: An iterative algorithm for system of generalized equilibrium problems and fixed point problem. Fixed Point Theory and Applications 2014, 2014:235

\section{Submit your manuscript to a SpringerOpen ${ }^{\odot}$ journal and benefit from:}

- Convenient online submission

- Rigorous peer review

- Immediate publication on acceptance

- Open access: articles freely available online

- High visibility within the field

- Retaining the copyright to your article 